\title{
MovableType Software for Fast Free Energy-Based Virtual Screening: Protocol Development, Deployment, Validation, and Assessment
}

\author{
Zheng Zheng, Oleg Y. Borbulevych, Hao Liu, Jianpeng Deng, Roger I. Martin, and Lance M. Westerhoff*
}

Cite This: J. Chem. Inf. Model. 2020, 60, 5437-5456

Read Online

ABSTRACT: For decades, the complicated energy surfaces found in macromolecular protein:ligand structures, which require large amounts of computational time and resources for energy state sampling, have been an inherent obstacle to fast, routine free energy estimation in industrial drug discovery efforts. Beginning in 2013, the Merz research group addressed this cost with the introduction of a novel sampling methodology termed "Movable Type" (MT). Using numerical integration methods, the MT method reduces the computational expense for energy state sampling by independently calculating each atomic partition function from an initial molecular conformation in order to estimate the molecular free energy using ensembles of the atomic partition functions. In this work, we report a software package, the DivCon Discovery Suite with the MovableType module from QuantumBio Inc., that performs this MT free energy estimation protocol in a fast, fully encapsulated manner. We discuss the computational procedures and improvements to the original work, and we detail the corresponding settings for this software package. Finally, we

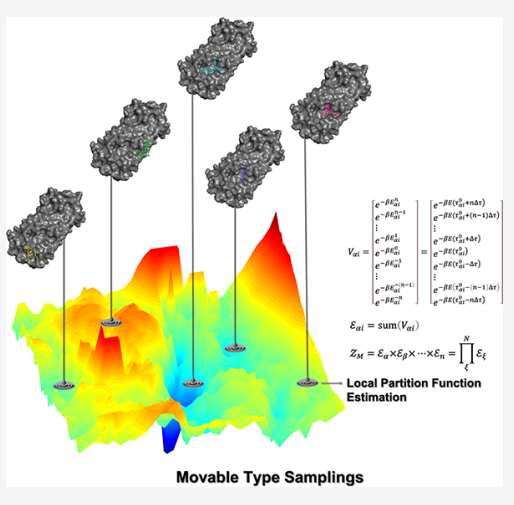
introduce two validation benchmarks to evaluate the overall robustness of the method against a broad range of protein:ligand structural cases. With these publicly available benchmarks, we show that the method can use a variety of input types and parameters and exhibits comparable predictability whether the method is presented with "expensive” X-ray structures or "inexpensively docked" theoretical models. We also explore some next steps for the method. The MovableType software is available at http://www.quantumbioinc.com/

\section{INTRODUCTION}

The cost of research and development in drug discovery has continued to increase annually, ${ }^{1,2}$ and much of this cost is due to the massive amount of screening for bioactive compounds required, in which only $1-2 \%$ of the screened lead compounds enter the preclinical stage. ${ }^{1}$ Receptor:ligand binding free energy simulation has become a vital research area in structure-based drug design, and accurate simulation of receptor:ligand free energy changes upon binding requires a thorough sampling of the metastable energy states on the dissociation pathway. Effective in silico predictions of the free energy changes with respect to biomolecular binding processes provide significant support to drug target identification and drug candidate screening and greatly reduce the cost of the corresponding "wet chemistry" research.

For several decades, on account of their speed and lower cost versus both molecular dynamics (MD) simulations and "wet chemistry" approaches, virtual screening and docking/ scoring methods have been applied to drug discovery. These methods have become integral to the drug discovery effort, as they are critical to understanding intermolecular interactions in the structure-based drug discovery effort. ${ }^{3-10}$ However, they are often criticized for their lack of accuracy in predicting binding modes and binding affinities, especially for the noncomparability of the scores to the experimental $\mathrm{p} K_{\mathrm{d}}$ values or free energies. ${ }^{11-16}$ Furthermore, predictions of smallmolecule docking often outperform those for larger molecules. ${ }^{17}$ Much of the challenge of docking/scoring is centered on the inability of these methods to sample enough of the relevant conformational space of the receptor:ligand complex. $^{18-21}$ Furthermore, the methods are often unable to correctly capture and sample structural water, ${ }^{22-24}$ tautomeric states, ${ }^{25,26}$ and conformational strain. ${ }^{27}$ These problems, coupled with scoring function errors ${ }^{28,29}$ and inaccurate protein:ligand complex structures, ${ }^{30,31}$ contribute to significant problems with the use of these methods in industrial drug discovery efforts. In order to decrease computational expense, protein flexibility is ignored ${ }^{32-34}$ and binding energy is approximated using "rigid receptor" or "induced-fit receptor" models, which use protein target minimization or refinement during the docking/scoring process. ${ }^{34}$ On the other hand,

Special Issue: Novel Directions in Free Energy

Methods and Applications

Received: June 2, 2020

Published: August 13, 2020

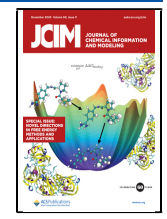


molecular simulation methods like $\mathrm{FEP}+{ }^{35}$ AMBER TI (thermodynamic integration), ${ }^{36,37}$ molecular mechanics/Poisson-Boltzmann surface area (MM/PBSA) and molecular mechanics/generalized Born surface area (MM/GBSA), ${ }^{38-42}$ linear interaction energy (LIE), ${ }^{43}$ and replica exchange with solute tempering (REST), ${ }^{44,45}$ which are generally computationally expensive for large-scale virtual screening campaigns, are becoming more accessible and easier to use with the general availability of graphics processing unit (GPU) technologies. These methods can effectively simulate receptor-ligand binding/dissociation trajectories and are in theory better able to predict free-energy-based binding affinities. Free energy perturbation or alchemical methods have shown promise, ${ }^{8,18,46-48}$ while absolute free energy determination is still a problem and these methods often exhibit significant errors. ${ }^{18,49-54}$ Free energy algorithms that effectively balance speed and accuracy are in high demand according to the growing need for accurate computational methods in the fastpaced drug discovery and biotechnology industries.

Beginning in 2013, Merz and co-workers developed ${ }^{55}$ and patented $^{56}$ the Movable Type (MT) free energy method to address this speed versus accuracy issue through the use of fast numerical integration methods to estimate the atomic energystate ensembles in the vicinity of one or more user-provided or automatically generated structural state(s). These atom-level ensembles are grouped into molecular energy ensemble calculations in order to estimate the free energy of binding in a statistical-mechanically rigorous fashion. Over the years, the MT method has been expanded and refined to account for greater protein structure flexibility, ${ }^{57,58}$ ligand flexibility, ${ }^{59}$ a new atom:atom pair potential, ${ }^{60}$ and the KMTISM molecular solvation model. ${ }^{61}$ Recently, in collaboration first with the Merz research group at Michigan State University and then with the Zheng research group at Wuhan University of Technology and building off our previous efforts in computational chemistry,9,10,62 and X-ray crystallography, ${ }^{63-65}$ we reimplemented the MT method in an package for deployment in industrial/commercial pharmaceutical research and drug discovery. This implementation has expanded on the original approach through greater speed and stability, improved usability, integration with third-party software packages and graphical user interfaces for execution of standard virtual screening protocols, and support for additional "built-in" and "user-supplied" atom:atom pair potentials in order to support more chemical environments. In present work, we report this MT free energy estimation implementation using this new software package through the treatment of two validation benchmarks: (1) the industry-standard Comparative Assessment of Scoring Functions (CASF-2016) set, which contains 57 protein targets and 285 ligands, was utilized to validate the robustness of the MT protocol across a broad range of protein classes, and (2) a set of 10 protein targets with a total of 248 ligands was selected from the PDBBind database in order to further explore MT performance in virtual screening tasks targeting large-ligand structural diversity for individual receptors. This work primarily focused on validation of rigidand semirigid-receptor/flexible-ligand MT, which is likely better suited to structures that show smaller structural movements upon binding. However, in the last paragraphs of the paper, we discuss next steps with the method as we expand on its capabilities for greater receptor flexibility, including support for MD snapshots/trajectories, loop and rotamer sampling, and so on.

\section{METHODS}

Traditionally, configurational energy state sampling for a macromolecule (e.g., a protein or protein:ligand complex) is extremely computationally expensive because of the all-atom flexibility that must be employed. Coupling all-atom atom:atom pairwise interaction calculations with sampling results in a huge computational cost. It is not unusual for MD simulation methods-which are used for this purpose-to require hundreds or even thousands of CPU hours to complete, often relying on the use of specialized hardware like Anton ${ }^{66}$ or repurposed GPU cards ${ }^{67-69}$ to make the simulations more tractable for routine application. To address this molecular energy state sampling expense, the MT method employs the assumption that given a reasonable molecular sampling volume for an NVT ensemble, the molecular partition function can be approximated as the product of the atomic partition functions. The MT method therefore postulates that within a volume of motion, each atom possesses an independent potential energy distribution. The purpose of this approximation is to treat each of the sampled molecular energy states as an independent numerical integration for each atomic partition function in order to estimate the molecular free energy.

Numerical Integration of the Atomic Energy Ensembles. Using one or more end-state conformations of a receptor:ligand complex, for all of the atoms in the structure, the method samples an identical amount of motion by generating atom:atom pairwise Boltzmann factors using discrete pairwise distance values within a given range. Therefore, the energy of an atom (e.g., atom $\alpha$ ) is divided into pairwise interactions between atom $\alpha$ and each of the other atoms in the complex (e.g., atom $i$ ). In this model, the ensemble of $\alpha: i$ atom:atom pairwise energy states within that range is captured using the Boltzmann factor vector $V_{\alpha i}$ depicted in eq 1 :

$$
V_{\alpha i}=\left[\begin{array}{c}
v_{\alpha i}^{n} \\
v_{\alpha i}^{n-1} \\
\vdots \\
v_{\alpha i}^{1} \\
v_{\alpha i}^{0} \\
v_{\alpha i}^{-1} \\
\vdots \\
v_{\alpha i}^{-(n-1)} \\
v_{\alpha i}^{-n}
\end{array}\right]=\left[\begin{array}{c}
\mathrm{e}^{-\beta E_{\alpha i}^{n}} \\
\mathrm{e}^{-\beta E_{\alpha i}^{n-1}} \\
\vdots \\
\mathrm{e}^{-\beta E_{\alpha i}^{1}} \\
\mathrm{e}^{-\beta E_{\alpha i}^{0}} \\
\mathrm{e}^{-\beta E_{\alpha i}^{-1}} \\
\vdots \\
\mathrm{e}^{-\beta E_{\alpha i}^{-(n-1)}} \\
\mathrm{e}^{-\beta E_{\alpha i}^{-n}}
\end{array}\right]=\left[\begin{array}{c}
\mathrm{e}^{-\beta E\left(\tau_{\alpha i}^{0}+n \Delta \tau\right)} \\
\mathrm{e}^{-\beta E\left(\tau_{\alpha i}^{0}+(n-1) \Delta \tau\right)} \\
\vdots \\
\mathrm{e}^{-\beta E\left(\tau_{\alpha i}^{0}+\Delta \tau\right)} \\
\mathrm{e}^{-\beta E\left(\tau_{\alpha i}^{0}\right)} \\
\mathrm{e}^{-\beta E\left(\tau_{\alpha i}^{0}-\Delta \tau\right)} \\
\vdots \\
\mathrm{e}^{-\beta E\left(\tau_{\alpha i}^{0}-(n-1) \Delta \tau\right)} \\
\mathrm{e}^{-\beta E\left(\tau_{\alpha i}^{0}-n \Delta \tau\right)}
\end{array}\right]
$$

in which $\tau_{\alpha i}^{0}$ corresponds to the initial coordinates of atom pair $\alpha: i$ in the input structure (or structures) and $\Delta \tau$ represents a single unit or component of variation with a sampling range $( \pm n \Delta \tau)$ of $n$ discrete states. $V_{\alpha i}$ is a set of Boltzmann factors of atom pair $\alpha: i$, and for each pairwise contact including atom $\alpha$, these sets can be modeled as $V_{\alpha j}, V_{\alpha k}, V_{\alpha l}$, and so on.

The pairwise Boltzmann factors corresponding to the sampled atom:atom pairs in the structure are combined, leading to a local partition function for each atom $\alpha$ that contains a large number of energy states. It would be extremely time-consuming to generate all of the available states with respect to a single atom $\alpha$. Furthermore, this expense would be 


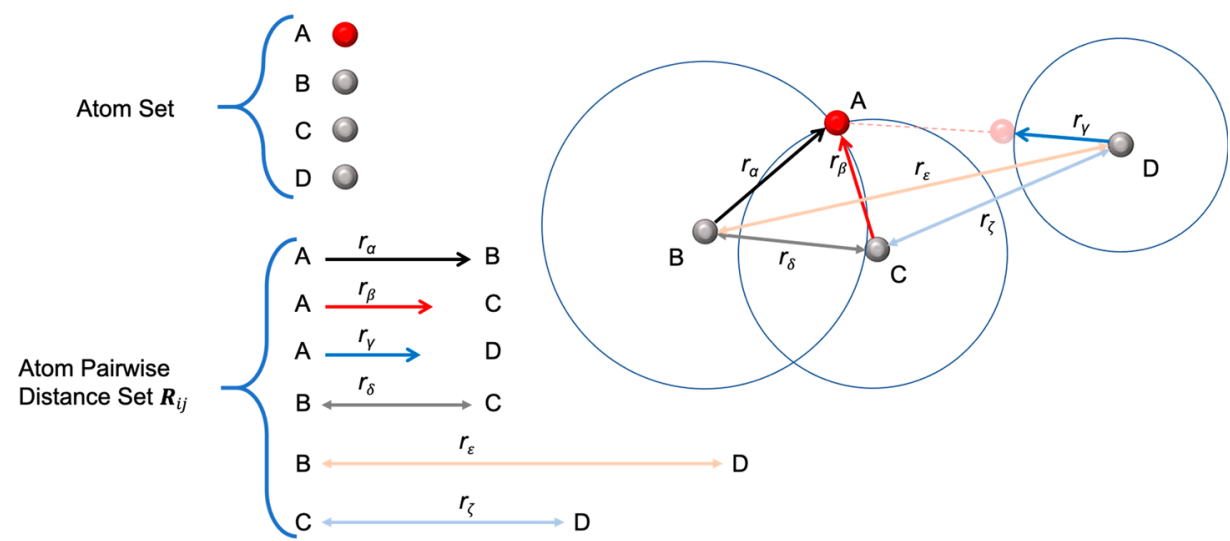

Figure 1. The number of atomic pairwise distance degrees of freedom of $\boldsymbol{R}_{i j}$ is dependent on the number of atomic coordinate degrees of freedom of $\boldsymbol{X}_{i}$. Assuming a four-atom molecular system, a group of randomly assigned atomic pairwise distances $R_{i j}$ may not be able to construct a valid 3-D structure. As shown in this figure, there is no location for atom A to satisfy $r_{\alpha}, r_{\beta}$, and $r_{\gamma}$ at the same time given the set $R_{i j}$.

compounded when all of the atoms in the molecule are likewise treated in order to calculate the overall molecular free energy. Instead, by the use of the following method, the Boltzmann factors for different atom:atom pairwise contacts are treated independently, and we calculate the local partition function for each atom without creating the entire set of configurations. Equation 2 depicts the sum of the energy states of atom pair $\alpha: i$,

$$
\begin{aligned}
\varepsilon_{\alpha i} & =\operatorname{sum}\left(V_{\alpha i}\right) \\
& =\left[\mathrm{e}^{-\beta E\left(\tau_{\alpha i}^{0}-n \Delta \tau\right)}+\ldots+\mathrm{e}^{-\beta E\left(\tau_{\alpha i}^{0}\right)}+\ldots+\mathrm{e}^{-\beta E\left(\tau_{\alpha i}^{0}+n \Delta \tau\right)}\right]
\end{aligned}
$$

and per the distributive property, multiplication of $\varepsilon_{\alpha i}$ and $\varepsilon_{\alpha j}$ yields the sum of all energy states for atom $\alpha$ combining $\alpha: i$ and $\alpha: j$ contacts (eq 3 ):

$$
\begin{aligned}
\varepsilon_{\alpha i} \times \varepsilon_{\alpha j}= & {\left[\mathrm{e}^{-\beta\left(E_{\alpha i}^{-n}+E_{\alpha j}^{-n}\right)}+\mathrm{e}^{-\beta\left(E_{\alpha i}^{-n}+E_{\alpha j}^{-(n-1)}\right)}+\ldots\right.} \\
& +\mathrm{e}^{-\beta\left(E_{\alpha i}^{-n}+E_{\alpha j}^{0}\right)}+\mathrm{e}^{-\beta\left(E_{\alpha i}^{-n}+E_{\alpha j}^{1}\right)}+\ldots \\
& +\mathrm{e}^{-\beta\left(E_{\alpha i}^{-n}+E_{\alpha j}^{n-1}\right)}+\mathrm{e}^{-\beta\left(E_{\alpha i}^{-n}+E_{\alpha j}^{n}\right)}+\ldots \\
& +\mathrm{e}^{-\beta\left(E_{\alpha i}^{0}+E_{\alpha j}^{-n}\right)}+\mathrm{e}^{-\beta\left(E_{\alpha i}^{0}+E_{\alpha j}^{-(n-1)}\right)}+\ldots \\
& +\mathrm{e}^{-\beta\left(E_{\alpha i}^{0}+E_{\alpha j}^{0}\right)}+\mathrm{e}^{-\beta\left(E_{\alpha i}^{0}+E_{\alpha j}^{1}\right)}+\ldots \\
& +\mathrm{e}^{-\beta\left(E_{\alpha i}^{0}+E_{\alpha j}^{n-1}\right)}+\mathrm{e}^{-\beta\left(E_{\alpha i}^{0}+E_{\alpha j}^{n}\right)}+\ldots \\
& +\mathrm{e}^{-\beta\left(E_{\alpha i}^{n}+E_{\alpha j}^{-n}\right)}+\mathrm{e}^{-\beta\left(E_{\alpha i}^{n}+E_{\alpha j}^{-(n-1)}\right)}+\ldots \\
& +\mathrm{e}^{-\beta\left(E_{\alpha i}^{n}+E_{\alpha j}^{0}\right)}+\mathrm{e}^{-\beta\left(E_{\alpha i}^{n}+E_{\alpha j}^{1}\right)}+\ldots \\
& \left.+\mathrm{e}^{-\beta\left(E_{\alpha i}^{n}+E_{\alpha j}^{n-1}\right)}+\mathrm{e}^{-\beta\left(E_{\alpha i}^{n}+E_{\alpha j}^{n}\right)}\right]
\end{aligned}
$$

When each atom:atom pairwise contact energy is treated independently, eq 3 represents all of the conformational energy states of atom $\alpha$ for a molecular system with atoms $\alpha, i$, and $j$. Calculation of the left-hand side of eq 3 through the multiplication of $\varepsilon_{\alpha i}$ and $\varepsilon_{\alpha j}$ saves the trouble (and time) of sampling all of the $(2 n+1)^{2}$ configurational states for the triatomic system. Following this procedure in a molecular system with $N$ atoms, multiplication of sums for $N-1$ pairwise contacts pertaining to atom $\alpha$ is performed, yielding a free energy ensemble of atom $\alpha$ for an $N$-atom molecular system including atom $\alpha$ (eq 4):

$$
\varepsilon_{\alpha}=\prod_{i}^{N-1} \varepsilon_{\alpha i}
$$

where $\varepsilon_{\alpha}$ is the local partition function within the range of motion between atom $\alpha$ and each of the atoms in the molecular system. Then, given the range of motion for each atom, the local partition functions for all of the atoms in the system are multiplied to generate the molecular energy-state ensemble (eq 5):

$$
\mathcal{Z}_{\mathrm{M}}=\varepsilon_{\alpha} \times \varepsilon_{\beta} \times \ldots \times \varepsilon_{n}=\prod_{\xi}^{N} \varepsilon_{\xi}
$$

Using eqs $1-5$, the MT calculation collects a molecular energy-state ensemble centered on an initial molecular conformation, combining term-by-term entries of all the atomic pairwise configurational vectors as in eq 1 .

Up to this point, we have applied a numerical protocol for fast estimation of the molecular local energy-state ensemble. However, such an approximation brings in a key source of error to the molecular free energy estimation: because an $\mathrm{N}$ particle system under the $3 N-6$ degrees of freedom does not support the random "mixing and matching" of particle pairwise distances of all the $N(N-1) / 2$ particle pairwise contacts in the system, unphysical energy states would be introduced into the $\mathcal{Z}_{\mathrm{M}}$ molecular partition function calculation (e.g., $\left.\varepsilon_{\alpha} \times \varepsilon_{\beta}\right)$. This situation is illustrated in Figure 1, which depicts an $N$ atom molecular system and a group of randomly selected atom pairwise distances that may not support a valid threedimensional (3-D) molecular structure. In this situation, the number of degrees of freedom of the atomic pairwise distance ensemble $\boldsymbol{R}_{i j}$ is dependent on the number of degrees of freedom of the atomic coordinate ensemble $\boldsymbol{X}_{i}$. In the following paragraphs, we use italic uppercase letters to represent a group of variables in which a variable vector $\left(e . g\right.$., $R_{i j}$ ) captures a certain set of atomic pairwise distances including all pairwise contacts in the molecular system. In this discussion, we use bold-italic uppercase letters to represent a group of variable vectors in which a vector ensemble (e.g., $\boldsymbol{R}_{i j}$ ) captures the ensemble of atomic pairwise distance sets in the molecular system.

Introducing the calculation protocol using eqs $1-5$ on the one hand significantly increases the speed for calculating the molecular energy ensemble $\mathcal{Z}_{\mathrm{M}}$. On the other hand, use of these equations introduces unphysical energy states into the molecular energy ensemble by including invalid $\boldsymbol{R}_{i j}$ sets. Therefore, the collection of Boltzmann factors, $V_{\alpha i}$, contains 
Given the atomic pairwise sampling range $V_{c}$ and the atom number $N$ of a closed system:

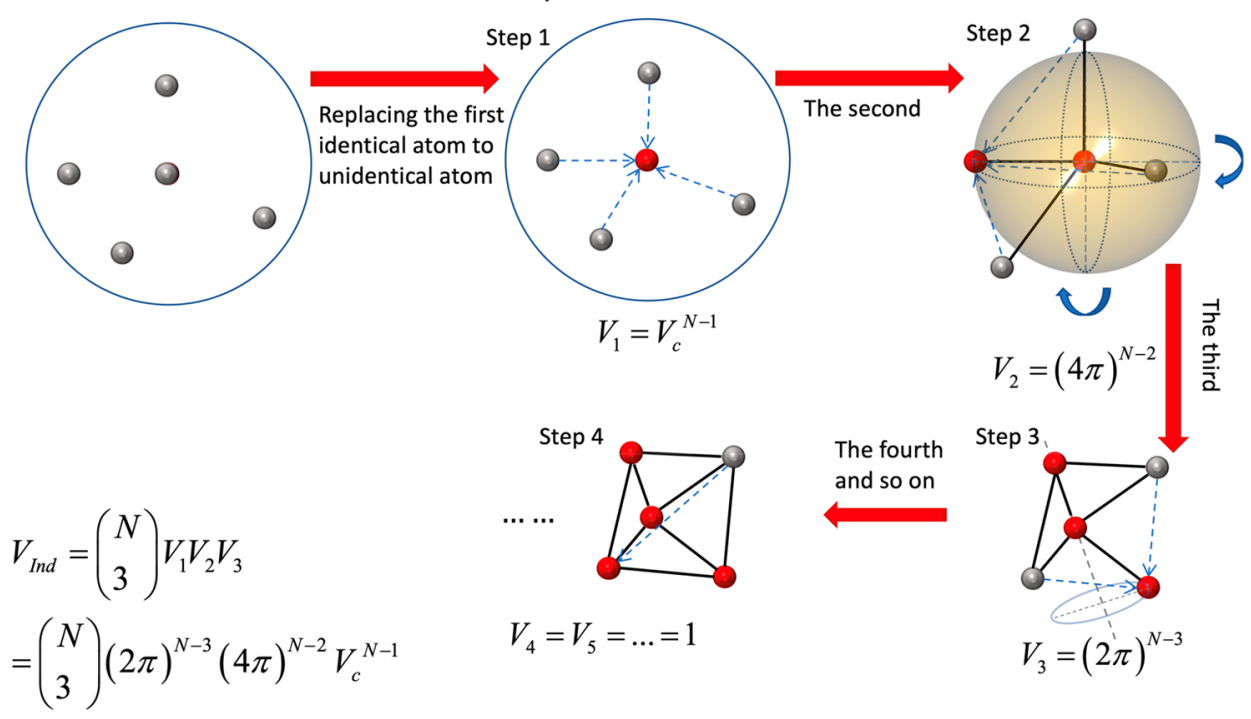

Figure 2. Illustration of the procedure for deriving $V_{\text {ind }}$, the number of degrees of freedom of the atomic pairwise contacts given the total number of atoms, $N$, and the common distribution range for all the atomic pairwise distances, $V_{c}$. For the example shown, a closed system with five atoms (with the blue circle as the volume boundary), the gray spheres represent the atoms in the background region with no pairwise contacts among them, and the red spheres represent the atoms in the explicit region for which the atomic pairwise contacts are taken into account. The blue dotted arrows represent new atomic pairwise contacts added to the system when one atom is moved from the background region to the explicit region. The black solid lines in each subfigure represent a set of atom pairwise contacts with certain combinations of pairwise distances selected from the degrees of freedom before a new atom is moved from the background region into the explicit region.

the $\pm n \Delta \tau$ sampling range shown in eq 1 , and the total number of energy states (including both physical and unphysical states) sampled in $\mathcal{Z}_{\mathrm{M}}$ is

$$
\mathrm{SS}=(2 n \Delta \tau)^{\mathrm{CN}}
$$

where $\mathrm{CN}$ is the total number of atomic pairwise contacts. We know that in molecular systems, the number of unphysical energy states increases as the sampling range $( \pm n \Delta \tau)$ grows. In order to address this error in the $\mathcal{Z}_{M}$ calculation, we studied the number of degrees of freedom of $\boldsymbol{R}_{i j}$ and compared it with the number of sampled energy states $(\mathrm{SS})$ in the numerical $\mathcal{Z}_{\mathrm{M}}$ calculation procedure. In this way, we selected a reasonable $V_{\alpha i}$ to balance the calculation accuracy and the sampling range of the molecular energy states.

Derivation of the Number of $\boldsymbol{R}_{i j}$ Degrees of Freedom, $\boldsymbol{V}_{\text {ind }}$. We applied the following procedure, summarized in Figure 2, for modeling $V_{\text {ind }}$ the number of $\boldsymbol{R}_{i j}$ degrees of freedom in an $\mathrm{N}$-atom molecular system. Consider an "alchemical" molecular model with $N$ atoms divided into two regions: (1) the explicit region, where each atom contacts all of the others, and (2) the background region, in which atoms come into contact only with the atoms in the explicit region and do not contact other background atoms. We place all of the atoms into the background region first and then move them one by one into the explicit region so that we can explain the modeling of the atom pairwise contact degrees of freedom in a step-by-step manner.

Step 1. When the first atom, $\alpha$, is placed into the explicit region and the other $N-1$ atoms are left in the background region, we have $N-1$ atom pairwise contacts all centered at atom $\alpha$. In this case, $V_{\text {ind }}$ can be modeled as $V_{\mathrm{c}}{ }^{N-1}$, where $V_{\mathrm{c}}$ is the distance distribution range of every $\alpha: i$ (explicit-atom:background-atom) atomic pairwise contact. According to the aforementioned MT sampling procedure, $V_{\mathrm{c}}$ is equal to the $\pm n \Delta \tau$ MT sampling range in eq 1 .

Step 2. When the second atom, $\beta$, is placed within the explicit region, $V_{\text {ind }}$ from step 1 is multiplied by $(4 \pi)^{N-2}$, meaning that on top of every molecular conformation generated in the first step (a set of $R_{\alpha-i}$ with certain combinations of $\alpha: i$ distances), the number of degrees of freedom increases by rotation of atom $\beta$ in a sphere centered at atom $\alpha$ when including the distance vector ensemble $\boldsymbol{R}_{\beta-j}$. Here $\boldsymbol{R}_{\beta-j}$ represents all possible combinations of the $\beta: j$ contact distances, where $j$ indicates any of the $N-2$ atoms in the background region at this stage. Hence, we have $V_{\text {ind }}=$ $V_{\mathrm{c}}^{N-1}(4 \pi)^{N-2}$ at this stage of the derivation.

Step 3. Similarly, when a third atom, $\gamma$, is moved into the explicit region, the number of degrees of freedom increases by a factor of $(2 \pi)^{N-3}$. Therefore, given a fixed set of $R_{\alpha-i}$ and $R_{\beta-j}$, both selected from the $V_{\mathrm{c}}^{N-1}(4 \pi)^{N-2}$ degrees of freedom, $2 \pi$ degrees of freedom are added for each $\gamma: k$ contact by letting atom $\gamma$ rotate around the axis defined by the vector from atom $\alpha$ to atom $\beta$, where $k$ indicates any of the $N-3$ atoms in the background region at this stage. This leads to $V_{\text {ind }}=$ $V_{\mathrm{c}}^{N-1}(4 \pi)^{N-2}(2 \pi)^{N-3}$.

Step 4. When a fourth atom, $\delta$, is moved into the explicit region and the new atom pairwise contacts regarding atom $\delta$ are taken into account, no extra degrees of freedom are added to $V_{\text {ind }}$. This is the case because on top of every set of $R_{\alpha-i}$, $R_{\beta-j}$, and $R_{\gamma-k}$, when any $\delta: l$ pairwise contacts are taken into account (where $l$ indicates any of the $N-4$ atoms left in the background region at this stage), no movement degrees of freedom for either atom $\delta$ or atom $l$ are allowed given a set of $\delta: \alpha, \delta: \beta$, and $\delta: \gamma$ distances and a set of $l: \alpha, l: \beta$, and $l: \gamma$ distances selected from the degrees of freedom modeled in steps $1-3$. From this point forward, no more degrees of freedom are added to $V_{\text {ind }}$ when new atoms are moved from the 

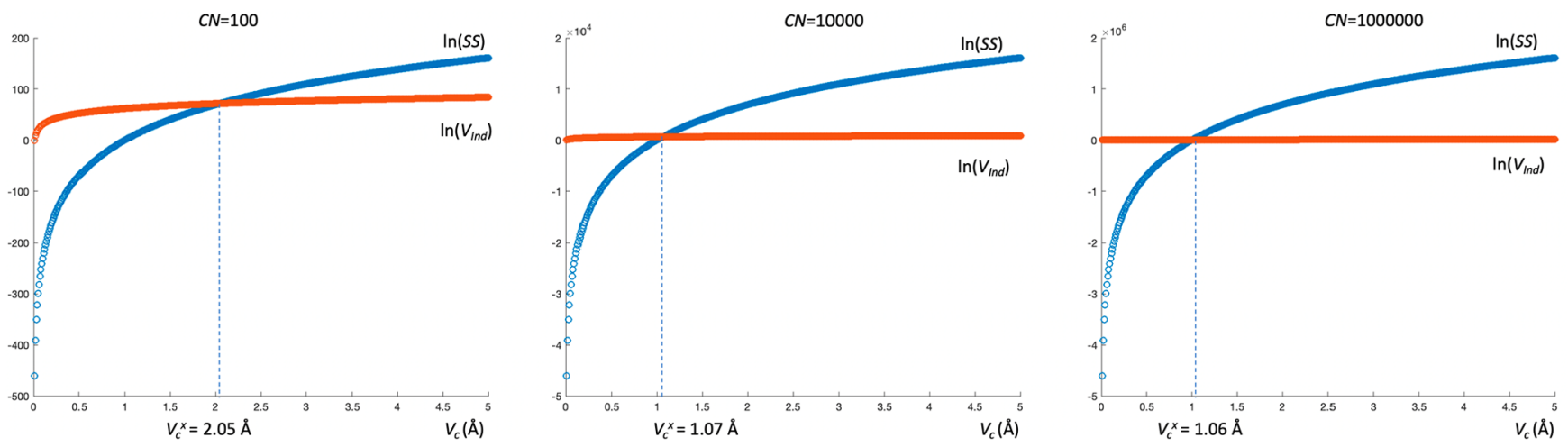

Figure 3. Distributions of $\ln (\mathrm{SS})$ and $\ln \left(V_{\text {ind }}\right)$ as functions of the atomic pairwise sampling range $V_{\mathrm{c}}$ given different $\mathrm{CN}$ values of the molecular system. The crossover point $V_{\mathrm{c}}^{x}$ approaches $1 \AA$ as $\mathrm{CN}$ increases.

background region to the explicit region. Therefore, no extra degrees of freedom of the atomic movement are allowed beyond the those included in $V_{\text {ind }}$ from steps $1-3$. Assuming equivalence among atoms with regard to the order of moving any three atoms into the explicit region, we complete the model by multiplying by the number of combinations of $\mathrm{N}$ atoms taken three at a time, as shown in eq 7 :

$$
V_{\text {ind }}=\left(\begin{array}{c}
N \\
3
\end{array}\right) V_{\mathrm{c}}^{N-1}(4 \pi)^{N-2}(2 \pi)^{N-3}
$$

In an $\mathrm{N}$-atom molecular system, the total number of atoms, $\mathrm{N}$, and the total number of atomic pairwise contacts, $\mathrm{CN}$, are mutually transformable using the following equations:

$$
\mathrm{CN}=\frac{N(N-1)}{2}
$$

and

$$
N=\frac{1+\sqrt{1+8 \times \mathrm{CN}}}{2}
$$

In the following procedure, we express $N$ in terms of $\mathrm{CN}$ using eq 9 and replace $2 n \Delta \tau$ with $V_{c}$ to make SS in eq 6 and $V_{\text {ind }}$ in eq 7 comparable, yielding eqs 10 and 11 :

$$
\begin{aligned}
\mathrm{SS}= & V_{\mathrm{c}}^{\mathrm{CN}} \\
V_{\text {ind }}= & \left(\begin{array}{c}
\frac{1+\sqrt{1+8 \times \mathrm{CN}}}{2} \\
3
\end{array}\right) V_{\mathrm{c}}^{[1+\sqrt{1+8 \times \mathrm{CN}} / 2]-1} \\
& (4 \pi)^{[1+\sqrt{1+8 \times \mathrm{CN}} / 2]-2}(2 \pi)^{[1+\sqrt{1+8 \times \mathrm{CN}} / 2]-3}
\end{aligned}
$$

Using eqs 10 and 11, we can compare the distribution of SS, i.e., the total sampled number of atomic pairwise contact energy states (including both physical and unphysical states), and the distribution of $V_{\text {ind }}$, i.e., the number of atomic pairwise contact degrees of freedom. Through this approach, we can determine a reasonable $V_{\mathrm{c}}$ to cover a fair range of molecular energy states in $\mathcal{Z}_{\mathrm{M}}$ and limit the number of unphysical states included in the $\mathcal{Z}_{\mathrm{M}}$ calculation. Since both SS and $V_{\text {ind }}$ grow exponentially as $V_{\mathrm{c}}$ increases, we use the logarithmic forms of their distributions in order to better compare them in Figure 3. Given a $\mathrm{CN}$ in the molecular system, $\ln (\mathrm{SS})$ grows faster than $\ln \left(V_{\text {ind }}\right)$ and soon surpasses it at the crossover point, $V_{c}^{x}$, as the atom:atom pairwise sampling range increases. For $V_{c}<V_{c}^{x}$, $\ln (\mathrm{SS})$ is smaller than $\ln \left(V_{\text {ind }}\right)$, showing that the number of sampled states from the MT procedure is smaller than the number of actual molecular energy states within the atom:atom pairwise sampling range. On the other hand, as the atom:atom pairwise sampling range increases beyond $V_{c}^{x}, \ln (\mathrm{SS})$ contains more states than $\ln \left(V_{\text {ind }}\right)$, and this is the point at which the MT procedure becomes contaminated by the unphysical states generated from the numerical integration. As depicted in Figure 3 , the crossover point, $V_{c}^{x}$, gradually approaches $1 \AA$ from $2.05 \AA$ as $C N$ increases from 100 to $10^{6}$. Therefore, in this study, we set the default MT atomic pairwise sampling range to $1 \AA$ for all calculations to avoid significant contamination of the free energy calculation by the introduction of unphysical states into the MT procedure. With a fixed $V_{c}$ for the atom:atom pairwise sampling range, SS for the number of MT sampled energy states, and $V_{\text {ind }}$ for the number of actual energy states, we applied a Monte Carlo integration to approximate the molecular local partition function:

$$
\mathcal{Z}_{\mathrm{M}}^{0} \approx V_{\mathrm{ind}} \frac{\mathcal{Z}_{\mathrm{M}}}{\mathrm{SS}}
$$

In summary, with the MT protocol, we utilize a sampling range $( \pm n \Delta \tau)$ for every atom:atom pair in a molecule or complex, and then we calculate an ensemble of atomic energy states using eqs $1-4$. The local partition function is then approximated first by combining these atomic energy ensembles using eq 5 and then by using the Monte Carlo integration procedure as shown in eq 12. Through this method, a local energy ensemble corresponding to a single initial "end-state" 3-D molecular conformation can be quickly calculated and converted into a local partition function.

In order to improve the method further, we know that free energy estimation relies on thorough molecular conformation sampling. Therefore, multiple end-state conformations can be provided to the MT method, where each end-state conformation can be viewed a representative or hypothetical landscape minimum, as discussed in the review by Mobley and Dill. ${ }^{21}$ This ensemble of poses is then combined to better capture larger-scale or "global" molecular movements. By feeding the MT protocol with multiple end-state conformations (i.e., $N_{\text {end-states }}$ ), the MT local partition function protocol can further enlarge the sampling space and better approximate the molecular partition function:

$$
\mathbb{Z}_{\mathrm{M}}=\sum_{\alpha}^{N_{\text {end-states }}} \mathcal{Z}_{\mathrm{M}}^{\alpha}=\mathcal{Z}_{\mathrm{M}}^{1}+\mathcal{Z}_{\mathrm{M}}^{2}+\ldots+\mathcal{Z}_{\mathrm{M}}^{N}
$$




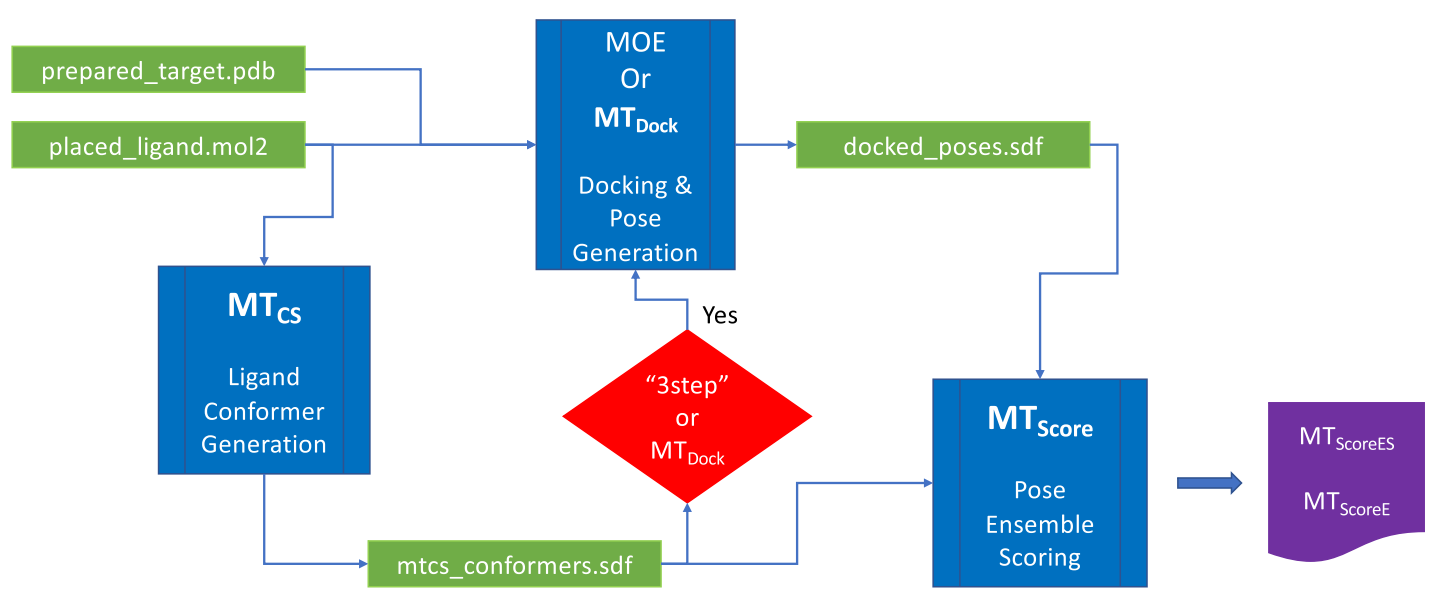

Figure 4. Overall flowchart of the MT method and its [optional] interactions with other software and methods. Generally, input is provided in the form of a prepared PDB and/or mol2 file for the target and ligand (a molecular selection language is provided in cases where these species are supplied in a single file). SDF files are used throughout to communicate docked poses or conformers as needed. Note: "nexus points" (shown in green) are provided for each MT step into which a user may optionally supply an externally prepared SDF file. These SDF files are only used when a third-party package such as MOE or GLIDE is used for docking and/or conformer generation. When $\mathrm{MT}_{\text {Dock }}$ is chosen as the docking function, all of the conformers and poses are communicated internally within the MT software and its associated data structures.

When applying the MT procedure to a protein:ligand complex system to estimate the binding free energy, we calculate partition functions for the bound-state protein:ligand complex and all of the unbound-state motifs. Each local partition function for the bound-state protein:ligand complex is calculated using the MT procedure (eqs 1-12) against the significant protein:ligand binding modes provided by executing the docking module from the software package or from the users' sources. By the use of eq 13, the protein:ligand complex partition function, $\mathbb{Z}_{\mathrm{PL}}$, is calculated as

$$
\mathbb{Z}_{\mathrm{PL}}=\sum_{\alpha}^{N_{\mathrm{PL}} \text { poses }} \mathcal{Z}_{\mathrm{PL}}^{\alpha}=\mathcal{Z}_{\mathrm{PL}}^{1}+\mathcal{Z}_{\mathrm{PL}}^{2}+\ldots+\mathcal{Z}_{\mathrm{PL}}^{N_{\mathrm{PL}} \text { poses }}
$$

In the present work, we added support for unbound- and bound-state structural motifs, including an apo protein conformation, multiple free-state ligand conformations, and multiple holo-protein:ligand conformations. Since a full-scale protein simulation requires significant computational cost, where noted we used induced-fit docking to collect multiple holo-protein:ligand conformations. Therefore, in addition to the calculation of $\mathbb{Z}_{\mathrm{PL}}$ with eq 14 , the protein local intramolecular partition function, $\mathbb{Z}_{\mathrm{P}}$, was calculated for a number of apo protein conformers, $N_{\mathrm{P} \text { conformers }}$ using eq 15 :

$$
\mathbb{Z}_{\mathrm{P}}=\sum_{\alpha}^{N_{\mathrm{P}} \text { conformers }} \mathcal{Z}_{\mathrm{P}}^{\alpha}=\mathcal{Z}_{\mathrm{P}}^{1}+\mathcal{Z}_{\mathrm{P}}^{2}+\ldots+\mathcal{Z}_{\mathrm{P}}^{N_{\mathrm{P} \text { conformers }}}
$$

where $N_{\mathrm{P} \text { conformers }}=1$ corresponds to the $\mathrm{X}$-ray model (sans ligand). With this technology available, in subsequent work we will explore the use of multiple X-ray, NMR, or theoretical models for both the apo protein and the holo-protein:ligand conformations. Finally, the free-state or unbound ligand conformations are generated using the small-molecule conformational search module, $\mathrm{MT}_{\mathrm{CS}}$, which is discussed in detail in a previous work. ${ }^{59} \mathrm{MT}_{\mathrm{CS}}$ constructs and characterizes $N_{\mathrm{L} \text { conformers }}$ ligand conformations, and the local partition functions for those ligand conformations are calculated and grouped using eq 16 :

$$
\mathbb{Z}_{\mathrm{L}}=\sum_{\alpha}^{N_{\mathrm{L}}^{\text {conformers }}} \mathcal{Z}_{\mathrm{L}}^{\alpha}=\mathcal{Z}_{\mathrm{L}}^{1}+\mathcal{Z}_{\mathrm{L}}^{2}+\ldots+\mathcal{Z}_{\mathrm{L}}^{N_{\mathrm{L} \text { conformers }}}
$$

With $\mathbb{Z}_{\mathrm{PL}}, \mathbb{Z}_{\mathrm{P}}$, and $\mathbb{Z}_{\mathrm{L}}$ available, the binding free energy change is then estimated using the ratio of partition functions in the bound and free states as per eq 17:

$$
\Delta G_{\text {binding }} \approx-R T \log \left(\frac{\mathbb{Z}_{\mathrm{PL}}}{\mathbb{Z}_{\mathrm{P}} \mathbb{Z}_{\mathrm{L}}}\right)
$$

The above-noted multiple-end-state protocol represented by eq 17 is denoted as $\mathrm{MT}_{\text {ScoreE}}$, in which the "E" denotes an ensemble of one or more end-state holo-protein:ligand conformations, apo protein conformations, and unbound ligand conformations. In addition to this more complete workflow, a simplified MT protocol was also implemented that uses a single end-state protein:ligand complex in a "minimum energy" conformation. Since this approach, which we name $\mathrm{MT}_{\text {ScoreES, }}$ where "ES" denotes the calculation against a single end-state protein:ligand 3-D complex, is based on a single accurate conformation and does not require docking or other simulation processes to generate, it is faster than $\mathrm{MT}_{\text {ScoreE }}$ and could therefore be better positioned for higher-throughput virtual screening tasks. Because $\mathrm{MT}_{\text {ScoreES }}$ utilizes only the intermolecular atom:atom pairwise potential calculation between the protein and the ligand, the binding free energy is then approximated as

$$
\Delta G_{\text {binding }} \approx-R T \log \left(\mathcal{Z}_{\text {InterPL }}^{0}\right)
$$

where $\mathcal{Z}_{\text {InterPL }}^{0}$ is the protein:ligand complex pose's local partition function considering only the intermolecular atomic pairwise interactions.

Ligand Binding Mode Preparation and Scoring. In the DivCon Discovery Suite v.DEV.671-b4608, we provide two empirical energy functions: the GARF statistical potential ${ }^{70}$ and the AMBERff14 functional potential ${ }^{63,71}$ optimized for the MT method. The holo-protein:ligand complex binding modes can be either generated using the "built-in" $\mathrm{MT}_{\text {Dock }}$ protein:ligand docking module ${ }^{55}$ or provided from other sources such as molecular simulations or alternative 
protein:ligand docking protocols. In order to compare the MT protocol performances with different settings, we applied both the GARF potential function and the AMBERff14 force field for the partition function calculation, and we used both $\mathrm{MT}_{\text {Dock }}$ and the industry-standard Molecular Operating Environment (MOE) v.2019.0102 from Chemical Computing Group, Inc. to generate contrasting protein:ligand complex poses. For $\mathrm{MT}_{\text {Dock }}$ and optionally for the MOE interface (in the "three-step workflow" discussed below), ligand conformers were generated using $\mathrm{MT}_{\mathrm{CS}}{ }^{59}$ The $\mathrm{MT}_{\mathrm{CS}}$ method was used in all cases to calculate the unbound $\mathbb{Z}_{\mathrm{L}}$ partition function. Figure 4 depicts a flowchart to aid in understanding how the various MT parts work together (and with third-party methods) to complete and generate the MT scores.

$\mathrm{MT}_{\text {Dock }}$ Configuration. Beginning with the aforementioned $\mathrm{MT}_{\mathrm{CS}}$ conformers, each of the top five lowest-energy conformers was placed multiple times within the crystallographic X-ray structure using the heatmap-based $\mathrm{MT}_{\text {Dock }}$ method reported by Zheng et al. ${ }^{55}$ Each ligand binding mode was optimized within the active site using the torsion optimization method discussed by Fuhrmann et al., ${ }^{72}$ and the top 25 scored poses according to $\mathrm{MT}_{\text {ScoreES }}$ were kept for inclusion in the $\mathrm{MT}_{\text {Score }}$ calculation. All of the $\mathrm{MT}$ calculations were performed with DivCon Discovery Suite v.DEV.671-b4608 using default settings with a pocket size of $8.0 \AA$ around the ligand (union between all poses) and a nonbonded interaction cutoff of $11.0 \AA$ A. Both the MT-GARF (-h garf) and MT-AMBER (-h amberff14) pair potentials were considered for this study.

MOE Docking Configuration. The calculation of $\mathrm{MT}_{\text {ScoreE }}$ (the ensemble $\mathrm{MT}_{\text {Score }}$ ) can be performed using either internally docked poses from $\mathrm{MT}_{\text {Dock }}$ or externally provided ligand poses (e.g., in the case of rigid-receptor docking) or protein:ligand poses (e.g., in the case of inducedfit-receptor docking) generated by third-party software tools. In order to demonstrate the generalizability of the method, we focused on rigid-receptor and induced-fit-receptor docking as implemented in MOE v2019.0102 using the qbDockPair.svl Scientific Vector Language (SVL) script found in the DivCon Discovery Suite package. The AMBER10 potential coupled with atomic charges and ligand parameters calculated using extended Hückel theory (Amber10:EHT) as implemented in MOE was used for all of the MOE-based calculations. Beginning with each PDB protein:ligand complex, protons were added, and their positions were optimized using Protonate3D. ${ }^{73}$ The default Protonate3D settings of 7, 300 $\mathrm{K}$, and $0.1 \mathrm{~mol} / \mathrm{L}$ for $\mathrm{pH}$, temperature, and ion concentration (salt), respectively, were chosen, and all of the atoms were allowed to flip, so some His, Asn, and Gln residues may have "flipped" during the protonation process (see the Supporting Information for all of the prepared structures used in this paper). When this basic preparation was completed for each structure, docking was executed using both the rigid-receptor docking and induced-fit docking refinement protocols.

For the MOE-based workflow, input conformers were generated two different ways: (1) in the conventional "threestep" protocol, $\mathrm{MT}_{\mathrm{CS}}$-generated conformers were provided as input to the MOE docking function (i.e., $\mathrm{MT}_{\mathrm{CS}} \rightarrow \mathrm{MOE} \rightarrow$ $\left.\mathrm{MT}_{\text {ScoreE }}\right)$, and (2) in the new "two-step" protocol, MOE's built-in conformer generator was used (i.e., $\mathrm{MOE} \rightarrow \mathrm{MT}_{\mathrm{ScoreE}}$ ). The three-step protocol uses $\mathrm{MT}_{\mathrm{CS}}$ in order to generate ligand conformers that exist on the ligand free energy surface with the chosen pair potential (e.g., MT-GARF or MT-AMBER) and to calculate the unbound $\mathbb{Z}_{\mathrm{L}}$ partition function. The five most energetically favorable conformers were chosen and passed to the MOE docker, which docks each conformer semirigidly (some in-dock optimization is performed, but bond rotations and rotamer flips are kept to a minimum). In selecting between these two conformer generation methods (two-step vs threestep), the benefit of the three-step method is that ligand poses are guaranteed to exist on the energy surface. The drawback is that the docker is limited to the conformers generated by $\mathrm{MT}_{\mathrm{CS}}$ even if they do not properly fit the active site. The twostep protocol skips the initial $\mathrm{MT}_{\mathrm{CS}}$ step for conformer generation, and the docker's built-in method (or another method of the user's choosing) is used both to generate the conformers of interest and to dock those conformers in the active site. This mode may be more accommodating to alternative binding mode selection in cases where the bound ligand pose deviates significantly from the $\mathrm{MT}_{\mathrm{CS}}$ conformers. However, as we will show in the Results and Discussion, there are times when its prediction profile is inferior.

When conformers were generated-either internally within $\mathrm{MOE}$ or externally using $\mathrm{MT}_{\mathrm{CS}}$ - initial docking placement was performed using the Triangle Matcher approach, and the London $\mathrm{dG}$ score and the generalized Born volume integral/ weighted surface area (GBVI/WSA) dG score function ${ }^{74}$ were used as the initial score and the final filter, respectively. The 250 poses provided by Triangle Matcher were optimized with the chosen refinement method (i.e., rigid-receptor/minimizedligand or induced-fit-receptor/minimized-ligand) using AMBER10:EHT as implemented in MOE, and 25 poses were finally passed to $\mathrm{MT}_{\text {ScoreE }}$ as landscape minima for scoring. All of the MT calculations were performed using DivCon Discovery Suite v.DEV.671-b4608 using default settings with a pocket size of $8.0 \AA$ around the ligand (union between all poses) and a nonbonded interaction cutoff of $11.0 \AA$. Both the MT-GARF (-h garf) and MT-AMBER (-h amberff14) pair potentials were considered.

Leave-One-Out Analysis. Leave-one-out cross-validation (LOO) is the statistical cross-validation method that leaves one data point (an observation) out of the data set and calculates the fit on the rest of the data in order to generate a prediction for the observed point. This process is repeated $n$ times, where $n$ is equal to the number of ligands in each target set, leading to $n$ predictions. Each time the omitted value, $y_{i 0}$, is predicted, the predicted residual, $\varepsilon_{i}=y_{i}-y_{i 0}$, is computed. Likewise, the mean unsigned error (MUE) is computed from the predicted residuals according to eq 19:

$$
\text { MUE }=\frac{\sum_{i}^{n}\left|y_{i}-y_{i 0}\right|}{n}
$$

The reported mean Pearson $R$ value is calculated according to eq 20 and is a result of this process since by definition with $n$ correlations we are able to calculate $n$ values of $R$ :

$$
R=\frac{\sum_{i}^{n} R_{i}}{n}
$$

Finally, the error bars in the figures and reported in the tables are constructed as vertical or horizontal lines defined for each point in the range $[R-\operatorname{MAD}(R), R+\operatorname{MAD}(R)]$ (for $R$ plots) or $[\mathrm{MUE}-\mathrm{MAD}(\mathrm{MUE}), \mathrm{MUE}+\mathrm{MAD}(\mathrm{MUE})]$ (for MUE plots). Instead of using the standard deviation (SD) to represent the spread of the data, we employ the median 

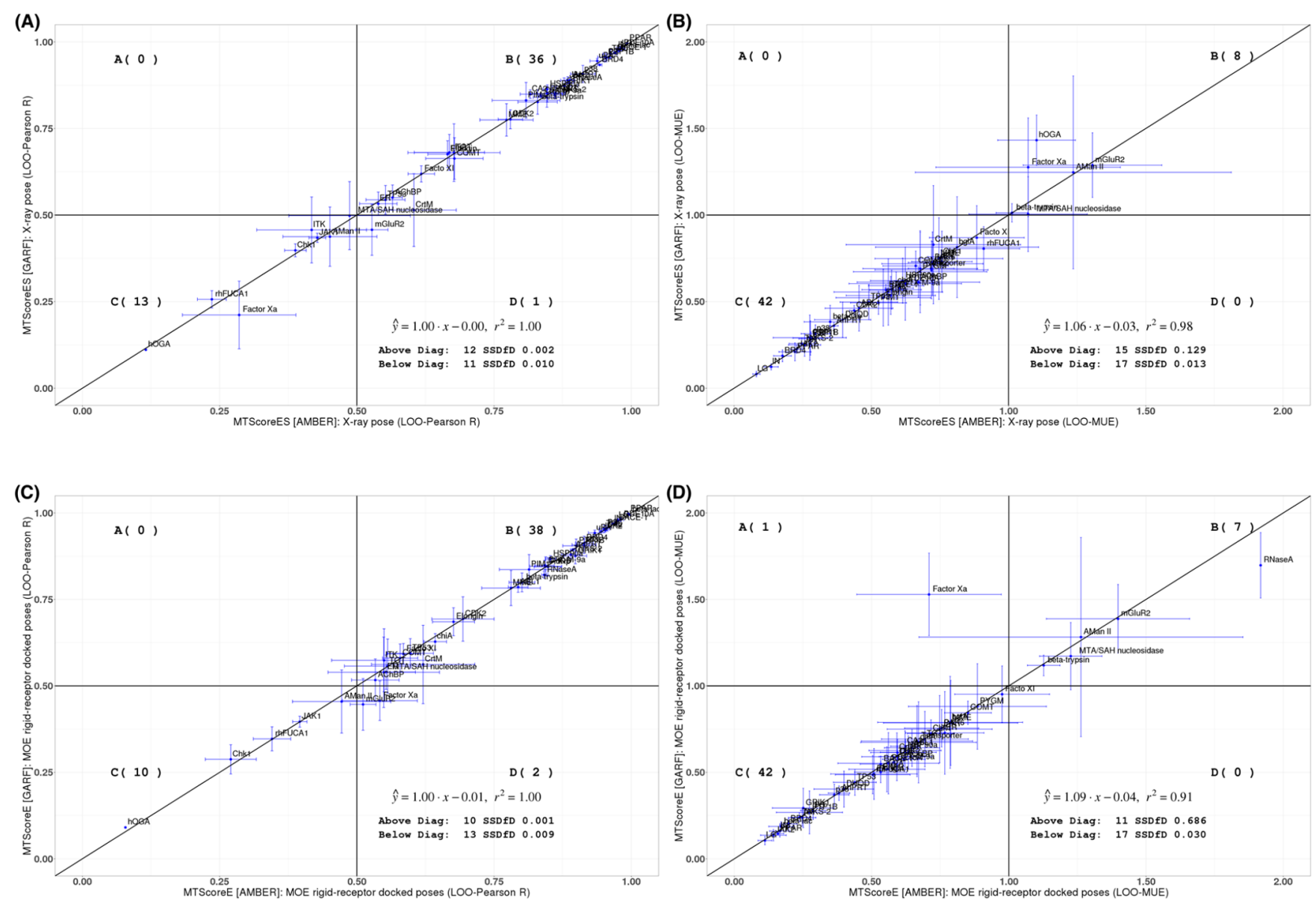

Figure 5. Comparison of Pearson $R$ values and LOO MUEs between the GARF and AMBERff14 energy functions using MT $_{\text {ScoreE }}$ (ensemble scoring with $\mathrm{MOE}$ rigid-receptor/minimized-ligand docked poses) and $\mathrm{MT}_{\text {ScoreES }}$ (end-state scoring with X-ray poses) depicting general agreement between the two methods. (A) Pearson $R$ values for the AMBERff14 and GARF energy functions through the $\mathrm{MT}_{\text {ScoreES }}$ calculation. (B) MUE values for both potential functions through the $\mathrm{MT}_{\text {ScoreES }}$ calculation. (C) Pearson R values for the AMBERff14 and GARF energy functions using the $\mathrm{MT}_{\text {ScoreE }}$ protocol. (D) MUE values for the AMBERff14 and GARF energy functions using the $\mathrm{MT}_{\text {ScoreE }}$ protocol. Table 1 provides a detailed numerical rundown of all cases.

absolute deviation (MAD), ${ }^{75}$ which is a robust measure of the spread of data around the median:

$$
\operatorname{MAD}=\operatorname{median}\left\{\left|X_{i}-\operatorname{median}(\mathbf{X})\right|\right\}
$$

where $X_{i}$ represents a data point and $\mathbf{X}$ is the array of data. The diagonal on the graph is defined as a line that passes through the points $(0,0)$ and $(1,1)$. The distance from the diagonal (DfD) for the point $P(x, y)$ is defined as

$$
\mathrm{DfD}=\frac{y-x}{\sqrt{2}}
$$

If $\mathrm{DfD}>0$, then the point is above the diagonal. Conversely, if $\mathrm{DfD}<0$, the point is below the diagonal. The squared sum of $\mathrm{DfD}$ (SSDfD), given by

$$
\mathrm{SSDfD}=\sum \mathrm{DfD}^{2}
$$

is computed separately for points above and below the diagonal and is a quantitative measure of such a deviation for the set.

\section{RESULTS AND DISCUSSION}

We utilized two validation sets to challenge the MT method for its robustness against a broad range of protein:ligand complexes and to test its consistency against different configurational state sampling protocols at various stages of the MT free energy estimation workflow. The first set consisted of the Comparative Assessment of Scoring Functions (CASF) protein:ligand scoring benchmark containing 57 protein targets with 285 ligands, which was first introduced with large diversity for both ligand and protein structures. ${ }^{76}$ The second set consisted of 10 protein targets with 248 corresponding ligands selected from the PDBBind ${ }^{77}$ v2019 database to study the performance of the MT protocol for screening of different ligand structures against particular receptors.

Comparative Assessment of Scoring Functions: The CASF-2016 Benchmark. The CASF benchmark consists of 57 target classes with five X-ray crystallographic structures for each target, yielding 285 target:ligand pairs. While there are some recognized deficiencies with some CASF-selected X-ray models, as a whole the set provides a reasonable cross section of the types of chemistry often observed in pharmaceutical research, and it has become an "industry standard" benchmark. Some curation was performed prior to commencement of the project. Specifically, since macrocycles are not supported by the method at this time, target cases that include macrocyclic ligands were removed. Likewise, cases that include large ligands (with more than 25 rotatable bonds) were removed 

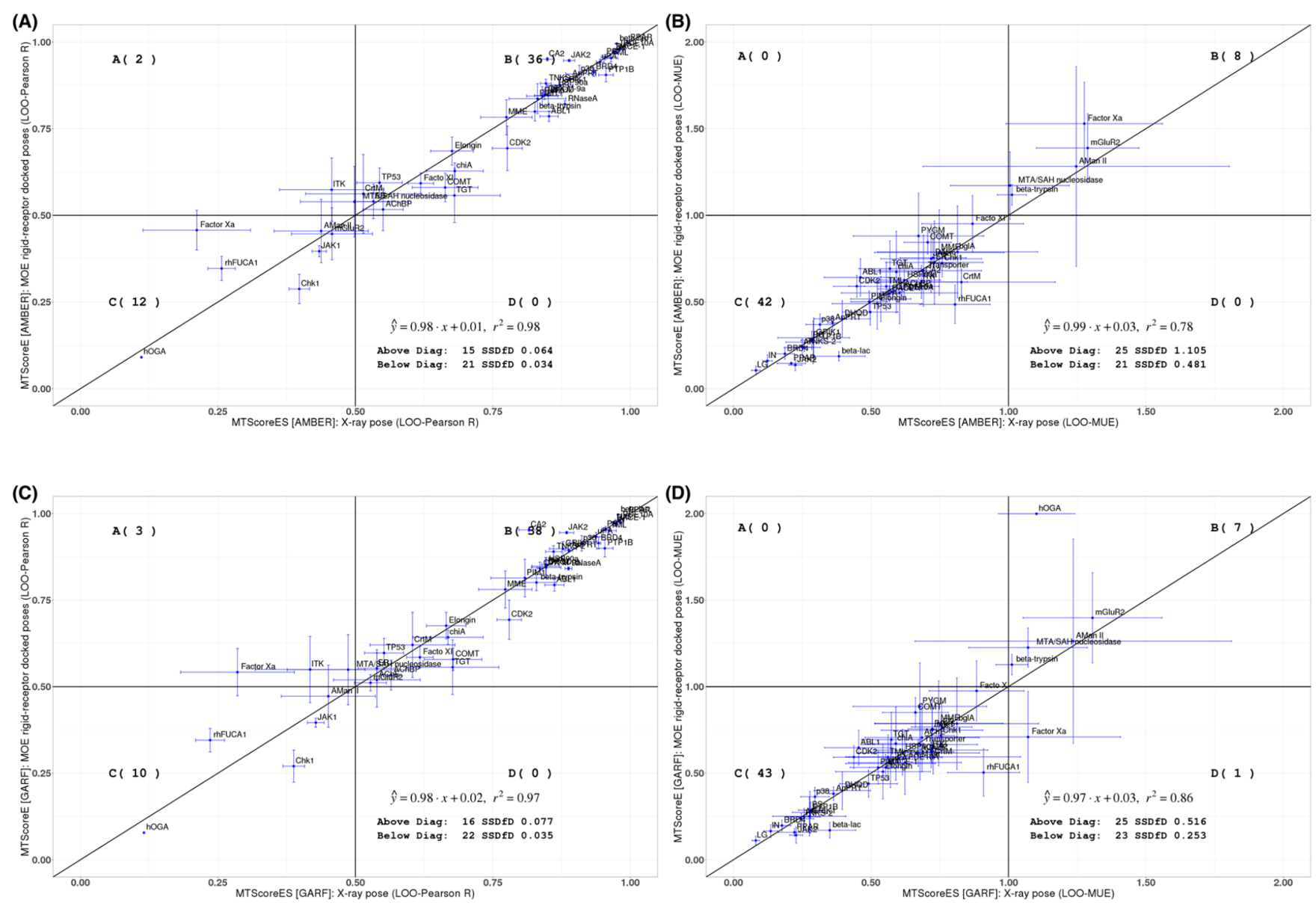

Figure 6. Comparison of Pearson $R$ values and MUEs between $\mathrm{MT}_{\mathrm{ScoreE}}$ (ensemble scoring with MOE rigid-receptor/minimized-ligand docked poses) and $\mathrm{MT}_{\text {ScoreES }}$ (end-state scoring with X-ray poses), in which we see convergence between the approaches. (A) Pearson $R$ values and (B) MUE values for the AMBERff14 energy function through the $\mathrm{MT}_{\text {ScoreES }}$ and $\mathrm{MT}_{\text {ScoreE }}$ calculations. (C) Pearson $R$ values and (D) MUE values for the GARF energy function through the $\mathrm{MT}_{\text {ScoreES }}$ and $\mathrm{MT}_{\text {ScoreE }}$ calculations. Table 1 provides a detailed numerical rundown of all cases.

from the set. This curation yielded 51 complete protein target class subsets $(5 \times 51=255$ structures $)$, and an additional 20 structures were rescued from the remaining six sets to give a total of 275 out of 285 PDB structures. Figure 5 is provided as a baseline comparison of MT-GARF and MT-AMBER showing that the two pair potentials are equally predictive in these cases.

Comparison of $\mathrm{MT}_{\text {ScoreEs }}$ (End-State Score) and $\mathrm{MT}_{\text {ScoreE }}$ (Ensemble Score). Traditionally, when the drug discovery process is considered, a critical goal is the determination of the experimental binding affinity of one or more lead compounds. With structure-based drug discovery, we wish to do so ideally prior to synthesizing the compound in the laboratory. This relationship between structure and function necessarily creates a "chicken versus egg" conundrum since the only way to experimentally determine binding is to synthesize potential compounds that may never bind. Likewise, predictive methods generally require reasonable compound binding modes in order to predict binding free energies, and these predicted binding free energies can vary significantly depending on the accuracy of the binding mode. X-ray crystallography is often used once a compound has been synthesized in order to provide an understanding of how a compound binds within the active site so that we may use that knowledge to inform the search for new lead compounds. However, X-ray crystallography is not an inexpensive process, and in a perfect world one would like to obtain an accurate understanding of binding affinity with less expense. Since $\mathrm{MT}_{\text {ScoreE }}$ incorporates multiple binding modes, in the first validation we used the "two-step" $\left(\mathrm{MOE} \rightarrow \mathrm{MT}_{\text {ScoreE }}\right)$ rigidreceptor docking protocol. Upon completion of the MOEbased docking process, these new bound-ligand poses were scored with MOE's built-in GBVI/WSA dG score in order to choose the top 25 bound-ligand poses to pass to $\mathrm{MT}_{\text {ScoreE }}$ (which were provided in SDF format).

Because all of the compounds in CASF have published X-ray models, we are able to compare the ensemble score generated using the chosen docking method to the end-state score calculated using the X-ray pose. Figure 6 depicts the $\mathrm{MT}_{\text {ScoreE }}$ versus $\mathrm{MT}_{\text {ScoreES }}$ results from the CASF benchmark for both AMBERff14 and GARF (note: for clarity, Table 1 provides a detailed rundown of all of the Pearson $R$ and LOO MUE results from the CASF benchmark as a function of atom:atom pair potential, scoring routine, and pose generation method

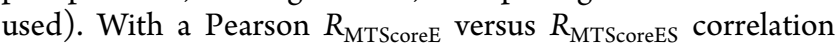
with $R^{2}>0.95$, we clearly observe that end-state scoring $\left(\mathrm{MT}_{\text {ScoreES }}\right)$ using crystal models as the input generally converges with ensemble scoring $\left(\mathrm{MT}_{\text {ScoreE }}\right)$ using the $\mathrm{MOE}$ docker with either potential function. These results suggest that given accurate structures, MT generally exhibits convergence between $\mathrm{MT}_{\text {ScoreE }}$ (with thorough computational sampling against the molecular configuration space) and 
Table 1. Detailed Comparison of the Predictive Capabilities of $\mathbf{M T}_{\text {ScoreES }}$ (End-State Score) and $\mathbf{M T}_{\text {ScoreE }}$ (Ensemble Score) and the Relative Predictive Capabilities of the Two Pair Potentials with Different Pose Generation Protocols

\begin{tabular}{|c|c|}
\hline 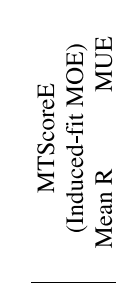 & 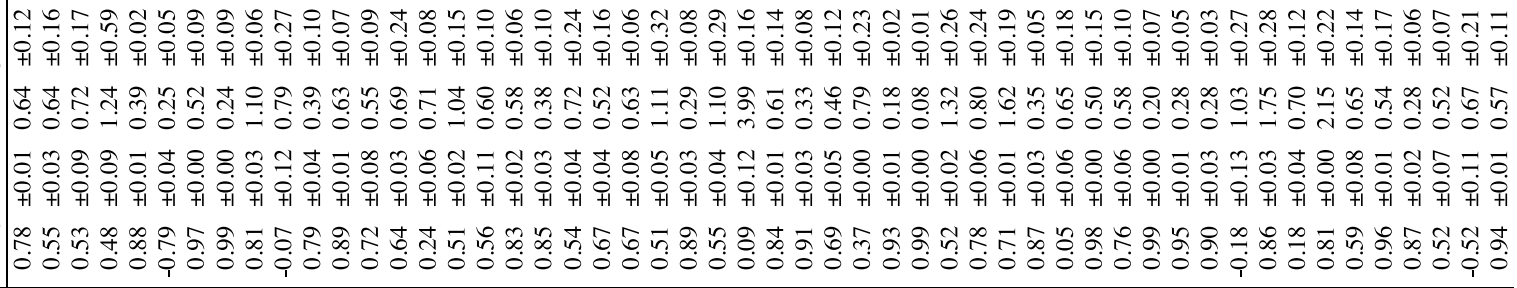 \\
\hline 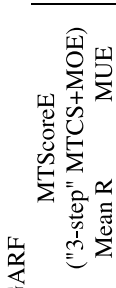 & 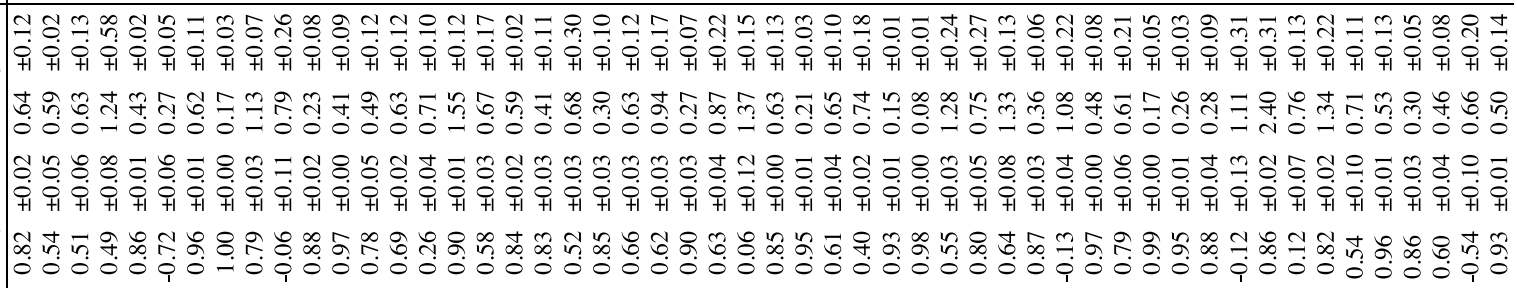 \\
\hline 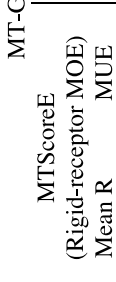 & 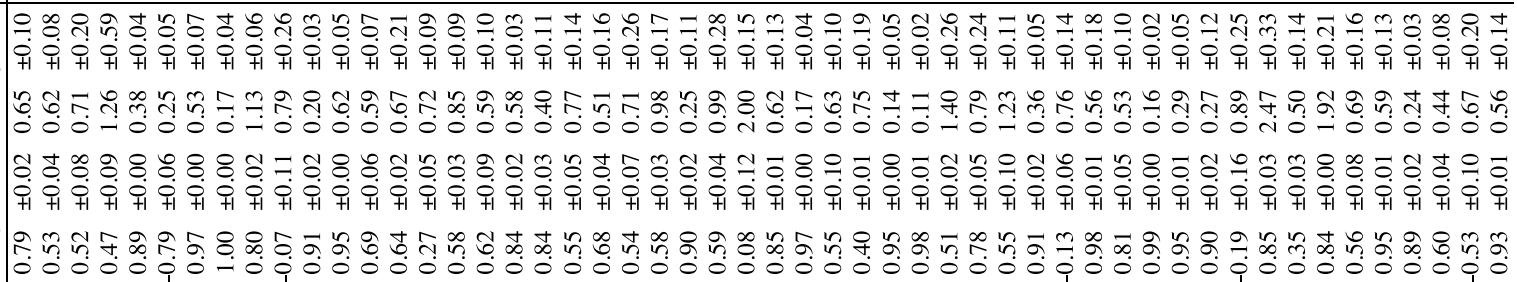 \\
\hline 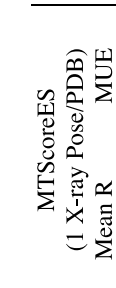 & 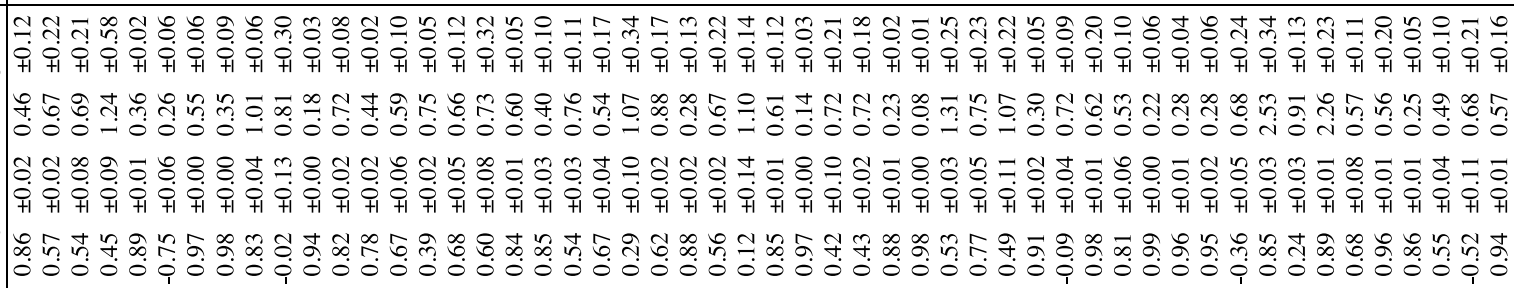 \\
\hline 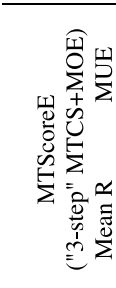 & 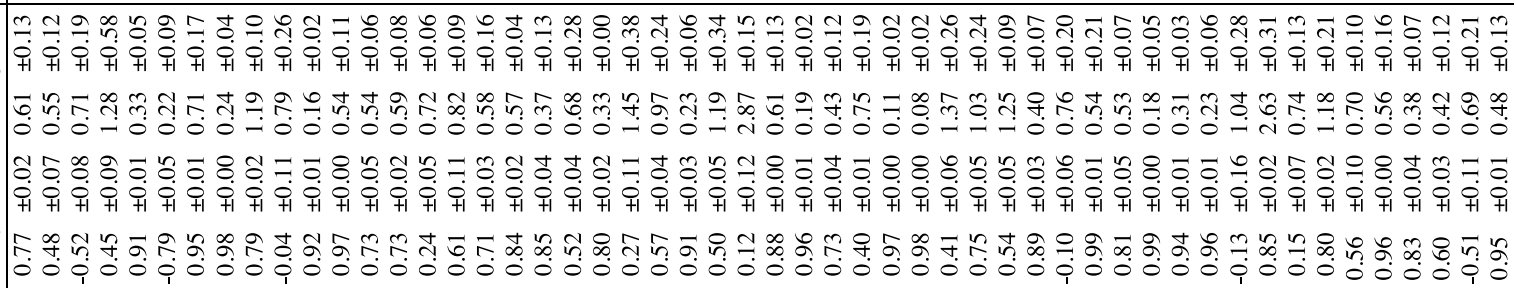 \\
\hline 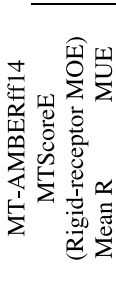 & 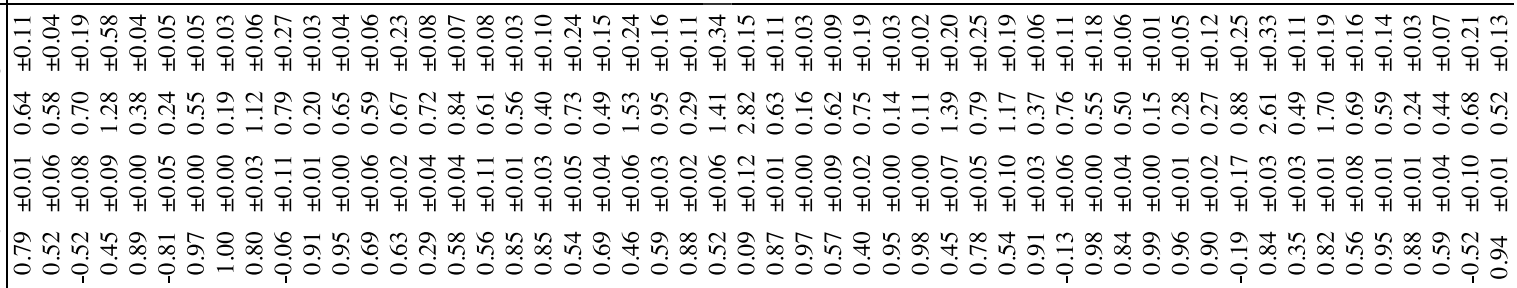 \\
\hline 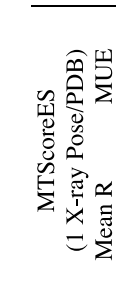 & 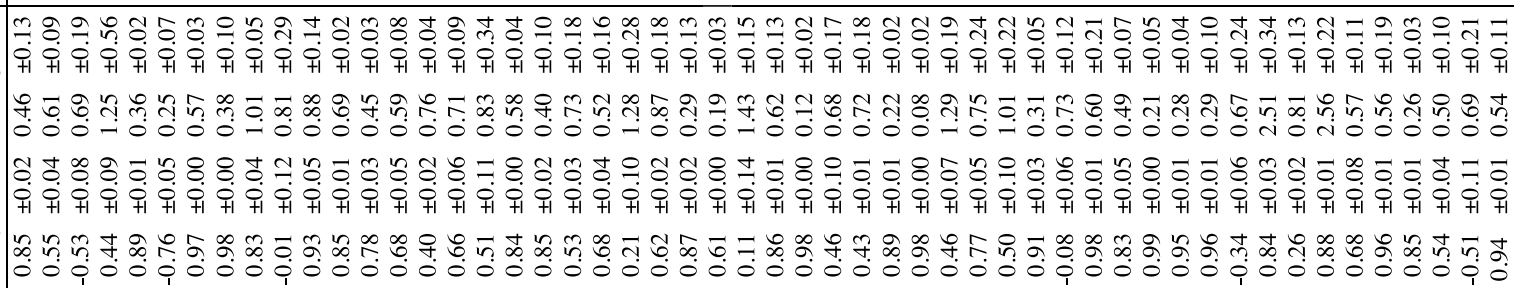 \\
\hline & 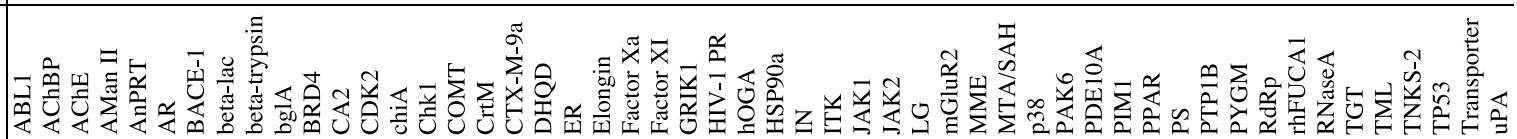 \\
\hline
\end{tabular}

${ }^{a}$ All of the MUE values are given in $\mathrm{kcal} / \mathrm{mol}$ and were obtained from the LOO analysis. 


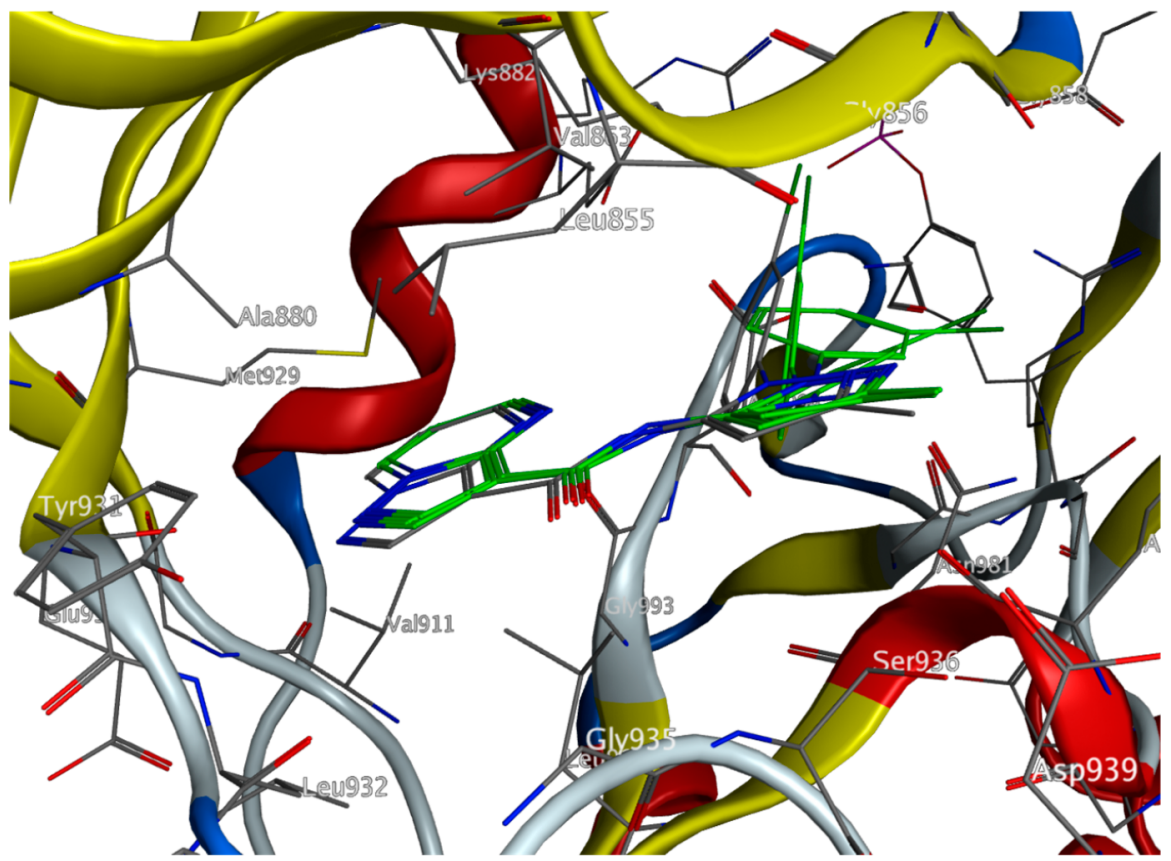

Figure 7. Example illustration of the top three scored poses (shown in green) and the X-ray pose (shown in default gray) within the active site of JAK2 kinase (PDB ID 4HGE) from the CASF set. The additional end-state (landscape minima) sampling provided by MOE-based rigid-receptor docking leads to an improved $\mathrm{MT}_{\text {ScoreE }}$ result vs the $\mathrm{MT}_{\text {ScoreES }}$ score of the original X-ray pose alone.

$\mathrm{MT}_{\text {ScoreES }}$ (with experimentally determined crystal poses). Furthermore, given the possibility that X-ray models (like any model) may be incorrect or may give an incomplete picture of the binding mode(s) available to the ligand, it is possible that $\mathrm{MT}_{\text {ScoreE }}$ is able to make up for deficiencies in the structure through the wider range of configurational sampling afforded by the ensemble score. An example of such a case is depicted in Figure 7. For JAK2 kinase (PDB ID 4HGE) from the CASF benchmark, the additional landscape minima (docked poses) provided by the MOE-based rigid-receptor/optimized-ligand docking routine led to the improved predicted versus experimental Pearson $R$ value observed in Figure 6C and detailed Table 1 for JAK2 for $\mathrm{MT}_{\text {ScoreE }}$ (Pearson $R_{\mathrm{MTScoreES}}=$ $0.88 \pm 0.01$ vs $\left.R_{\mathrm{MTS} \text { coreE }}=0.95 \pm 0.00\right)$. This observation fits with our expectation for free energy methods since we know that binding is a product of many poses and not just the one represented by a single crystal model. ${ }^{21}$

Comparison of the "Three-Step" and "Two-Step" $\mathrm{MT}_{\text {ScoreE }}$ Protocols. Next, we considered the impact of using MT-generated ligand conformers compared with using the conformers generated by the docking software (in this case $\mathrm{MOE})$. As discussed in Methods, $\mathrm{MT}_{\mathrm{CS}}$ generates an ensemble of ligand conformers to be used to determine the unboundligand partition function $\mathbb{Z}_{\mathrm{L}}{ }^{59}$ This step is performed regardless of how the ensemble score is calculated; however, one may choose to pass the top five (or more) $\mathrm{MT}_{\mathrm{CS}}$ unbound-ligand conformers to the docking function and use these conformers instead of those generated by the chosen docking function. For our analysis, we selected five conformers from $\mathrm{MT}_{\mathrm{CS}}$ in order to balance sampling thoroughness with efficiency. Since each conformer is used as an initial configuration for five binding modes $(5$ conformers $\times 5$ poses $=25$ binding modes), the computational time grows in $O(n)$ fashion as the number of conformers increases. Introducing additional conformers would cover more configurational space during binding mode sampling, but other than the significant states, additional sampled protein:ligand complex configurations contribute little to the final partition function. Table 2 shows that the impact of this choice is

Table 2. Impact of the Number of Chosen Conformers on the Overall Predictability of the Method

\begin{tabular}{cc} 
no. of conformers & overall Pearson $R$ \\
5 & 0.64 \\
10 & 0.64 \\
15 & 0.64 \\
20 & 0.63 \\
25 & 0.63 \\
\hline
\end{tabular}

generally small, and one can expect a limited return on one's investment for larger numbers of conformers. Likewise, as shown in Figure 8, when one considers the "best" conformer count for each target class versus the default count (5), the impact is relatively small with a few outlier cases.

The benefit of this approach is that the unbound-ligand conformations and the bound-ligand poses will not diverge appreciably from one another and will be within the same radius of convergence (since the docking process includes only placement and a localized structural minimization of the ligand within the field of the pocket). However, one could imagine some potential drawbacks of using these conformers, as there could be times when $\mathrm{MT}_{\mathrm{CS}}$ may choose conformers that will not "fit" the active site or there could be incompatibilities between the conformer generation algorithm and the chemistry of the ligand (i.e., every method has strengths and weaknesses, and often one may want to "mix and match" different conformer generators). Therefore, some dockers could be better able to generate conformers for the ligand chemistry in question. When the three-step $\left(\mathrm{MT}_{\mathrm{CS}} \rightarrow \mathrm{MOE} \rightarrow\right.$ $\mathrm{MT}_{\text {ScoreE }}$ ) workflow was executed on the CASF benchmark as depicted in Figure 9, these two approaches were also highly 


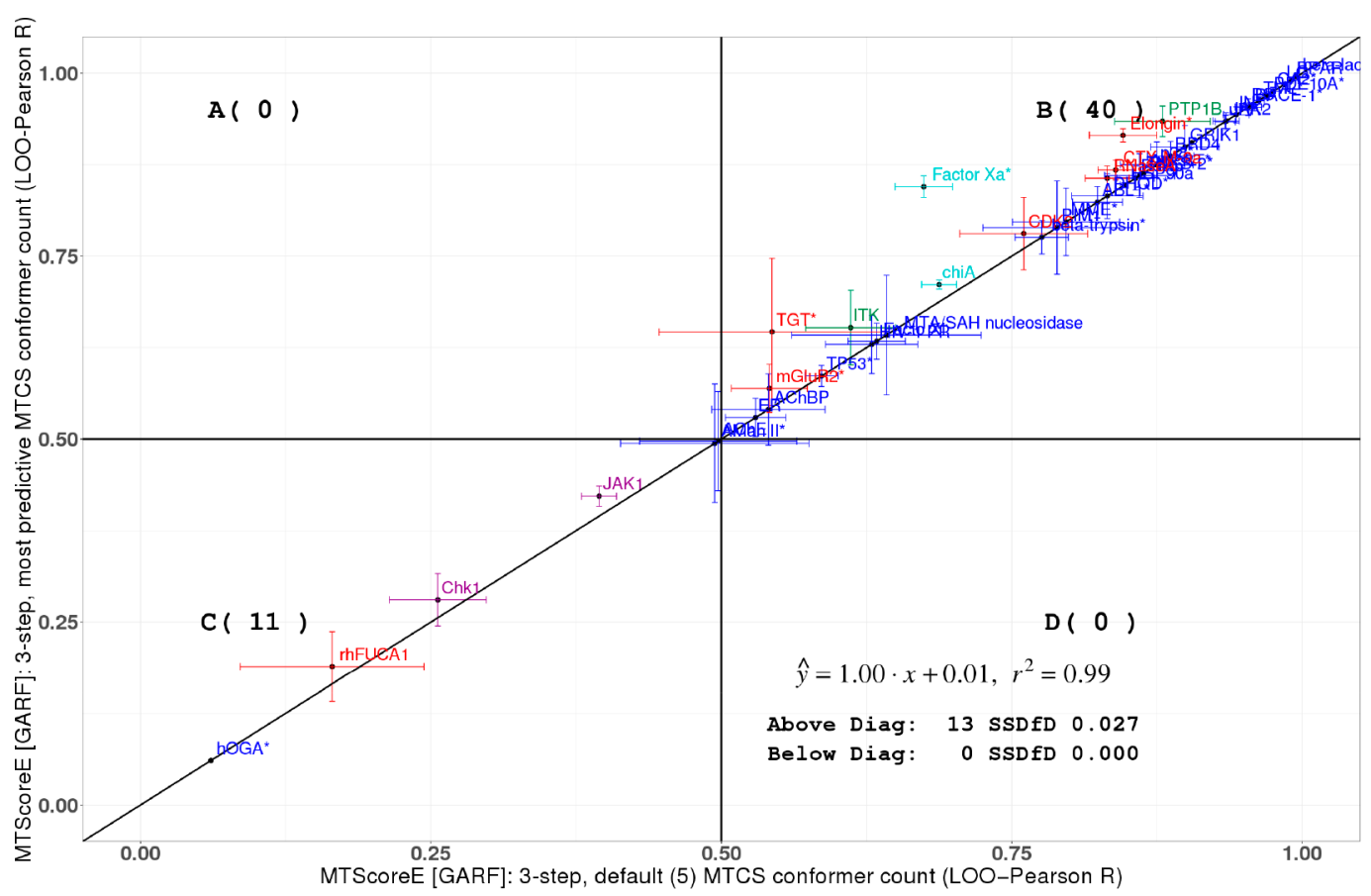

Figure 8. When exploring the impact of the $\mathrm{MT}_{\mathrm{CS}}$ conformer count in the "three-step" protocol, we consider the "best" conformer count vs the default conformer count (5) for each target class. The color of each target class corresponds to the minimum number of conformers needed to generate this best or most predictive set of scores. The classes in blue correspond to the default conformer count (5), while the red, cyan, magenta, and green classes correspond to calculations with 10, 15, 20, and 25 conformers, respectively.
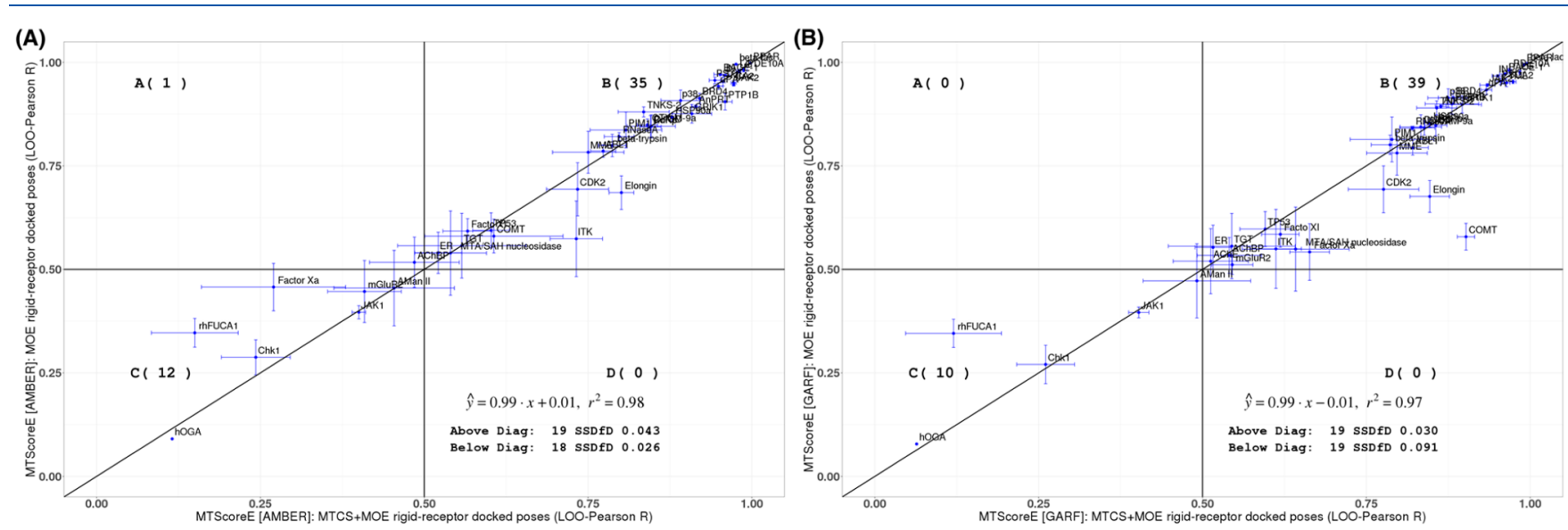

Figure 9. Comparison of the two-step $\left(\mathrm{MOE} \rightarrow \mathrm{MT}_{\mathrm{Score}}\right)$ and three-step $\left(\mathrm{MT}_{\mathrm{CS}} \rightarrow \mathrm{MOE} \rightarrow \mathrm{MT}_{\mathrm{Score}}\right)$ protocols, showing that generally these methods are highly correlated, with Pearson $R_{2 \text { step }}$ vs $R_{3 \text { step }}$ correlations with $R^{2}=0.98$ and 0.97 for (A) AMBERff14 and (B) GARF, respectively.

correlated, giving Pearson $R_{2 \text {-step }}$ versus $R_{3 \text {-step }}$ correlations with $R^{2}>0.95$ for both potentials. However, there are several outliers that make the three-step workflow worth considering in one's protocol (especially if the two-step workflow is not predictive enough for one's purposes). In particular, CDK2, elongin, and especially COMT (in GARF) and ITK (in AMBERff14) all yield better predictions (as measured by higher Pearson $R$ values) with the three-step protocol.

Impact of Induced-Fit-Receptor Docking on the Prediction Characteristics. For the GARF potential, intramolecular protein:protein interaction terms (eq 15) were added to the software in order to increase the accuracy of the MT approach and support a wider range of target structures and mechanisms. In order to explore the impact of these terms, we used induced-fit-receptor-docked ensembles as generated using the MOE platform. As depicted in Figure 10, while the deviations below the diagonal have a larger sum of squares than those above (suggesting that induced-fit-receptor docking is slightly less predictive than rigid-receptor docking in general), most of the shifts above or below the diagonal are quite small. However, there are several cases below the diagonal where induced-fit-receptor docking is more predictive. In these cases, as reported in Table 1, MTA/SAH went from $0.55 \pm 0.10$ in the rigid-receptor protocol to $0.71 \pm 0.01$ in the induced-fit-receptor approach, ITK went from $0.55 \pm$ 0.10 to $0.69 \pm 0.06$, and Factor Xa went from $0.54 \pm 0.07$ to $0.67 \pm 0.08$, suggesting that while the method generally shows a similar predictive profile, there are cases where the method is more predictive. Many reasons could cause an improvement in $R$ when a different docking strategy is used. One possible reason is the tight binding sites in these test cases brought difficulties for the rigid-receptor docking protocol in generating 

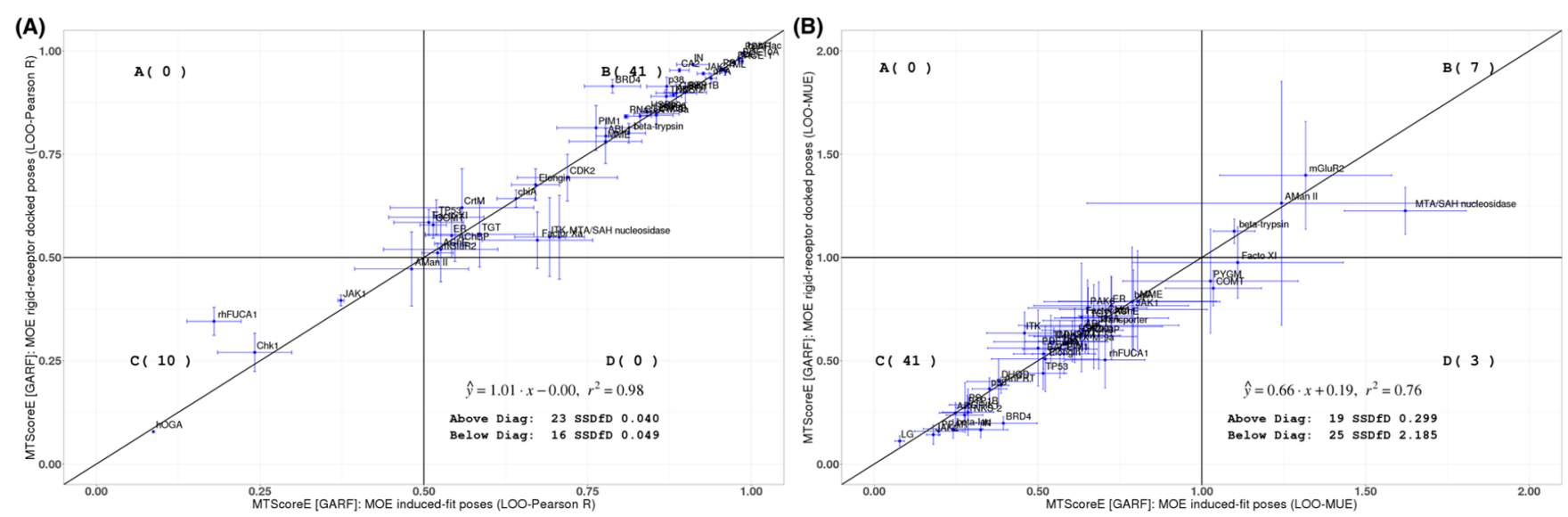

Figure 10. Comparison of (A) Pearson $R$ and (B) MUE values for rigid-receptor docking and induced-fit-receptor docking. The Pearson $R_{\text {rigid-receptor }}$ vs $R_{\text {induced-fit }}$ correlation has $R^{2}=0.98$, clearly indicating that the two methods are highly correlated, and one can generally rely on the lower-cost rigid-receptor method. Furthermore, most of the LOO MUEs are better than $1 \mathrm{kcal} / \mathrm{mol}$ for both methods.

the global-minimum bound states. When looking at the crystal complex structures, we found that all of the ligands for the MTA/SAH subset were closely surrounded by the binding site residues as in their binding modes. Furthermore, two test cases in the ITK subset (PDB IDs 4RFM and 4M0Z) were in a similar situation, and the ligands were tightly "wrapped up" by the surrounding receptor residues in the complex crystal structures. Finally, in the Factor Xa subset, ligands were placed deep into cavities at the receptor's binding site, where rigid docking strategies inevitably have difficulties fitting the ligand into the tight binding sites while avoiding steric clashes. These results are generally congruent with the literature, which has shown that induced-fit-receptor docking and induced-fitreceptor docking coupled with $\mathrm{MD}$ often yield improved predictions (versus rigid-receptor docking) for Factor Xa ${ }^{78,79}$ and ITK. ${ }^{80-82}$

Impact of Pose Count on the Results. Preparation of landscape minima is a critical aspect of the MT workflow. When poses provided by an external docking function (in this case MOE) are used, the success of the ensemble score is as much a function of the MT method as it is a function of the docker in question. Therefore, in order to explore the settings necessary to maximize performance, we ran several "set ranges", including $1-2,1-5,1-10,1-15,1-20$, and the default $1-25$, where the poses are ordered from best GBVI/ WSA dG score to worst (i.e., range 1-2 would include the top two poses according to MOE, range 1-5 would include the top five poses, and so on). These results are detailed in Table 3. Generally, for the CASF benchmark (with five ligands per target class) coupled with the MOE docking function, the $\mathrm{MT}_{\text {ScoreE }}$ method proved to be extremely robust, and often two well-scored poses (according to GBVI/WSA) were as good as 25 poses. This observation is very encouraging since it would suggest that most of the success of the method is driven by the local partition function. However, there are cases in which the addition of poses yields improved results. For example, BRD4 moved from a reasonably predictive $R^{2}$ of $0.64 \pm 0.05$ when the top two poses were scored to $R^{2}=0.94 \pm 0.01$ when the top 15 poses were included in the score. However, cases like CrtM, which went from $R^{2}=0.62 \pm 0.01$ when five poses were scored to $R^{2}=0.45 \pm 0.14$ when all 25 poses were scored, and MTA/SAH, which went from $R^{2}=0.51 \pm 0.08$ when five poses were scored to $R^{2}=0.34 \pm 0.10$ when all 25 poses were included, show that signal can be lost in the event that too many questionable poses are provided. This observation suggests that when the MT method is being challenged with a new project or target class, some retrospective experimentation with "knowns" may yield dividends when shifting to prospective campaigns.

Computational Time Requirements of the DivCon MT Implementation. When the MT method was first published, it was notable not only for its predictive capabilities but also for its economical use of CPU time versus methods that rely on $\mathrm{MD}$ or alchemical "webs" of MD calculations for sampling. Those earlier MT implementations were based on a mixture of MATLAB, Python, and bash scripts, and even at that time these calculations were considered to be fast. With the new DivCon Discovery Suite $(\mathrm{C}++)$ implementation of MT, we can quantify the average \pm MAD processor time on an older Intel Xeon E5440 $2.83 \mathrm{GHz}$ CPU running CentOS 7 for the 275 ligands in the CASF set, and we can break this time down into each step in the process: $1.0 \pm 0.0 \mathrm{~min} /$ ligand for $\mathrm{MT}_{\mathrm{CS}}$ $\left(\mathbb{Z}_{\mathrm{L}}\right.$ calculation and ligand conformer generation), $12.4 \pm 2.2$ $\mathrm{min} /$ ligand for MOE (rigid-receptor docking with ligand pose optimization), and $9.6 \pm 1.1 \mathrm{~min} /$ ligand for $\mathrm{MT}_{\text {Score }}\left(\mathbb{Z}_{\mathrm{PL}}\right.$ and $\mathbb{Z}_{\mathrm{P}}$ calculation using a 25 -pose ensemble). Since the calculation time for MT is measured in minutes on a standard CPU from 2008 and dynamics-based algorithms often require hours or even days to complete on specialized hardware (e.g., GPUs), the MT method would appear to be both economical and predictive.

The Homologous Protein Family (HPF) Benchmark. While the CASF benchmark was used to validate the MT method on a diverse set of targets, the Homologous Protein Family (HPF) benchmark introduced a series of homologous protein structures to demonstrate the performance of the MT method against a diverse set of ligands. As noted in Methods, both GARF and AMBERff14 atom:atom pair potentials were used, and two different docking programs (MOE and $\mathrm{MT}_{\text {Dock }}$ ) were considered. As listed in Table S1 in the Supporting Information, 10 homologous proteins with 248 corresponding ligands were selected from the PDBBind v2019 data set: 3dehydroquinate dehydratase (DHQD) with 22 ligands, 3phosphoinositide-dependent protein kinase-1 (PDPK1) with 26 ligands, 14-3-3 protein (14-3-3 $\eta$ ) with 12 ligands, acetylcholine receptor (AChR) with 38 ligands, $\alpha$-L-fucosidase (FUCA-1) with 12 ligands, $\beta$-glucosidase (GBA3) with 22 
Table 3. Impact of the Number of Poses Provided by MOE on the Predictive Capability of the MT ScoreE $_{\text {(Ensemble Scoring) }}$ Method

\begin{tabular}{|c|c|}
\hline & 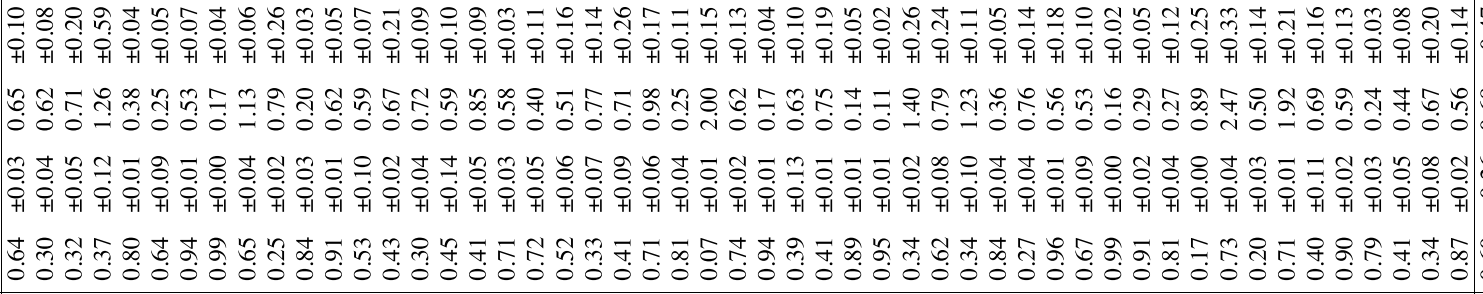 \\
\hline & 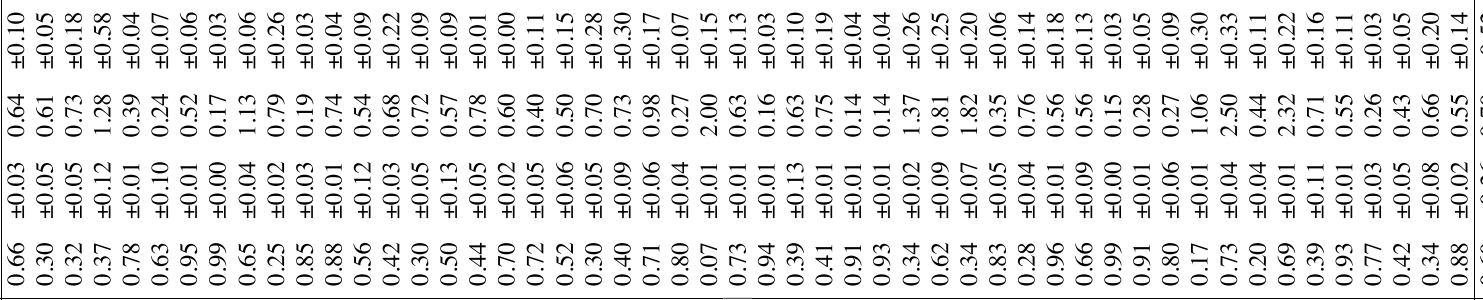 \\
\hline & 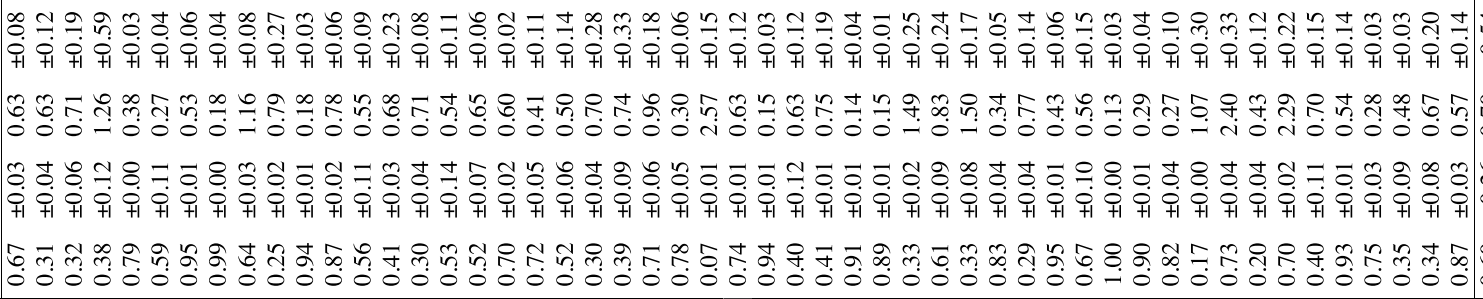 \\
\hline & 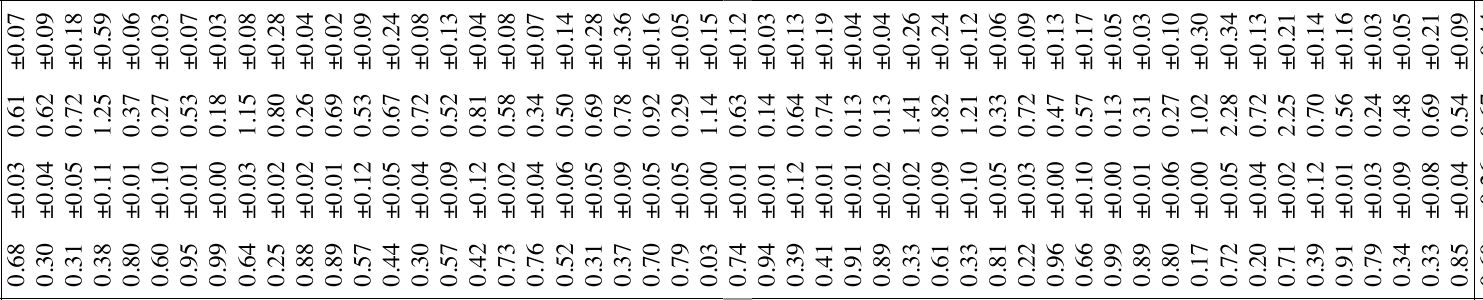 \\
\hline 3 & 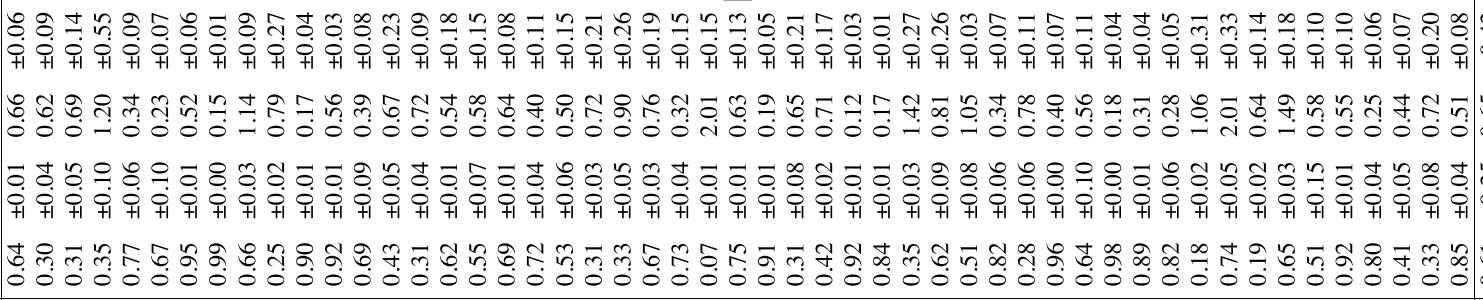 \\
\hline$\overline{\mathbb{s}}$ & 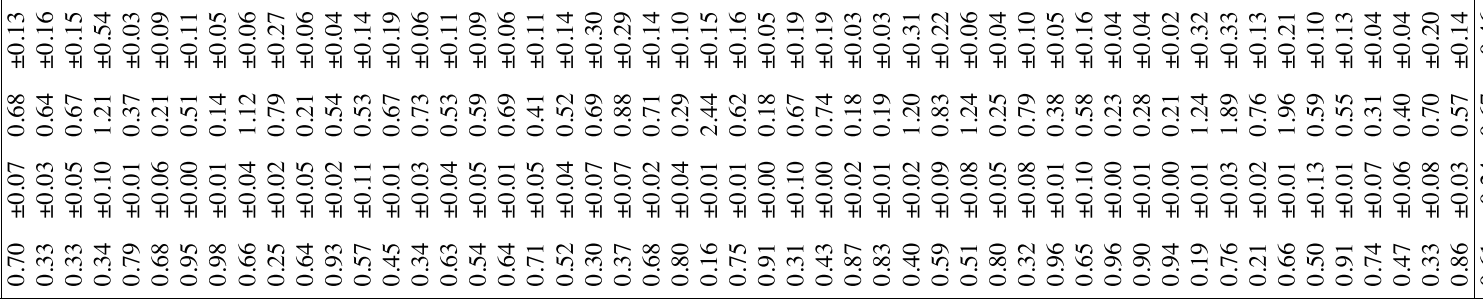 \\
\hline & 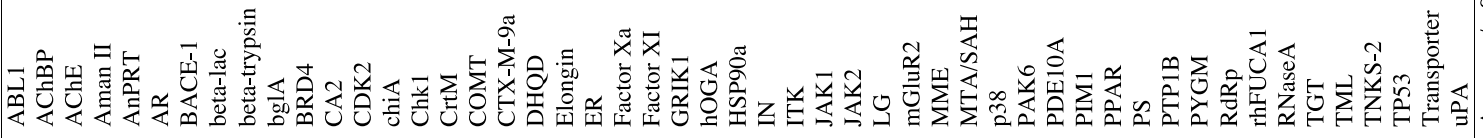 \\
\hline
\end{tabular}

${ }^{a}$ All of the MUE values are given in $\mathrm{kcal} / \mathrm{mol}$ and were obtained from the LOO analysis.

ligands, biotin carboxylase (BC) with 13 ligands, protein kinase A (PKA) with 47 ligands, trypsin (Tryp) with 20 ligands, and dual-specificity phosphatase (DSP) with 36 ligands. In choosing the protein:ligand structures available in the PDBBind v2019 set, ligands having a molecular masses of $<700 \mathrm{Da}$ and macrocyclic structures, against which the current version of the $\mathrm{MT}_{\mathrm{CS}}$ program cannot perform conformational search, were skipped. As with the CASF benchmark, we used the protein:ligand conformations from the crystal structures as the end-state input structures for the $\mathrm{MT}_{\mathrm{ScoreES}}$ calculations, and for $\mathrm{MT}_{\text {ScoreE }}$ the MOE-based two-step $\left(\mathrm{MOE} \rightarrow \mathrm{MT}_{\text {ScoreE }}\right)$ 
Table 4. Values of Pearson $R$ and MUE between the Experimental and Predicted Binding $\Delta G$ Values for $M_{\text {ScoreEs }}$ and $\mathbf{M T}_{\text {Score }}$ Calculations Performed with the DivCon Discovery Suite with the MovableType (MT) Module with Configurational Energies Evaluated Using the AMBERff14 and GARF Energy Functions

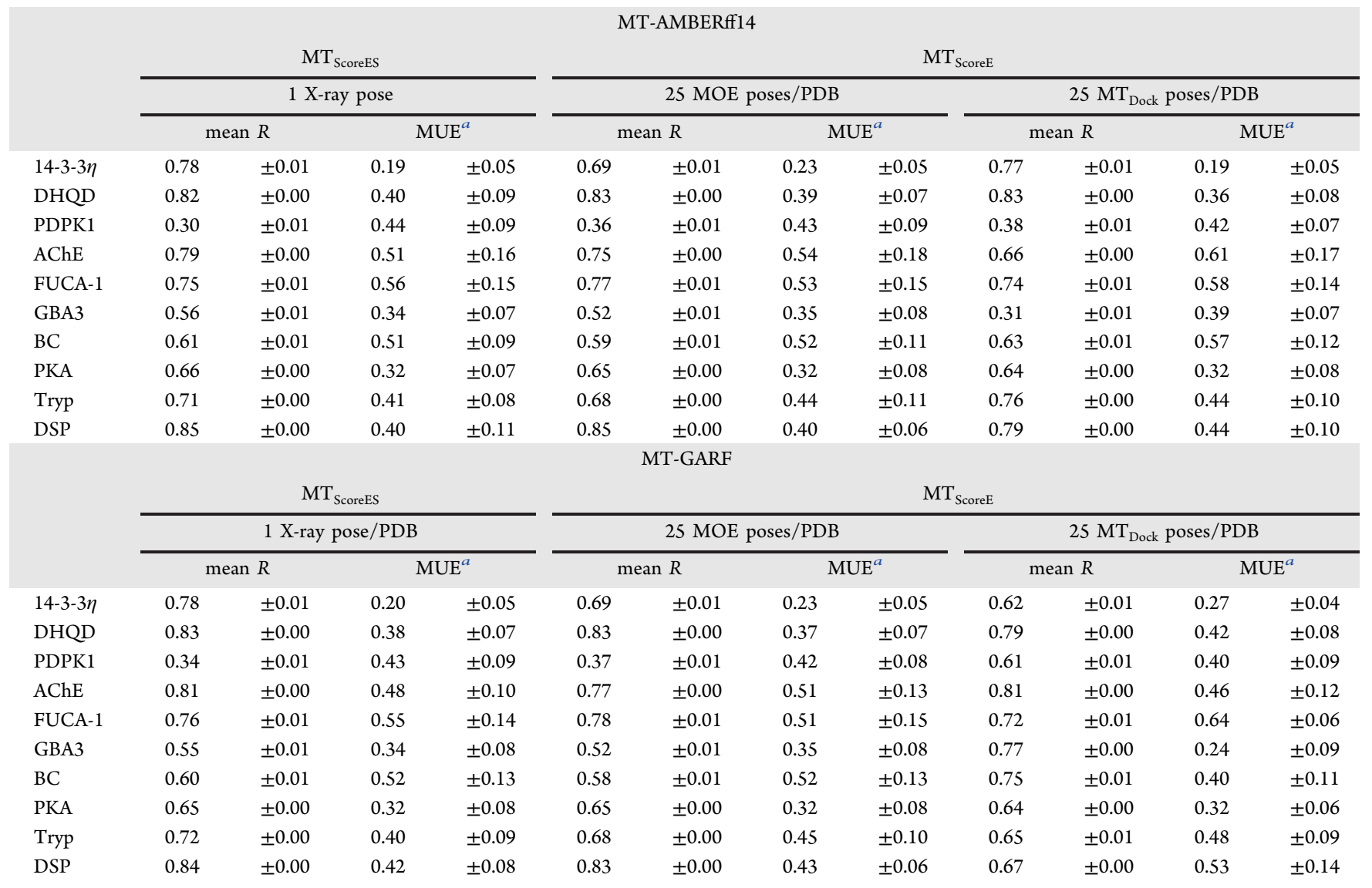

${ }^{a}$ All of the MUE values are given in $\mathrm{kcal} / \mathrm{mol}$ and were obtained from the LOO analysis.

protocol and the $\mathrm{MT}_{\text {Dock }}$ protocol $\left(\mathrm{MT}_{\mathrm{CS}} \rightarrow \mathrm{MT}_{\text {Dock }} \rightarrow\right.$ $\left.\mathrm{MT}_{\text {ScoreE }}\right)$ were compared and contrasted.

Using the $\mathrm{MT}_{\text {ScoreES }}$ protocol on the X-ray protein:ligand pose for each structure, both the AMBERff14 force field and the GARF energy function generated good correlations with the experimental binding affinities: the Pearson $R$ coefficients for both functions were higher than 0.5 for all of the protein sets except for PDPK1, which exhibited Pearson $R$ values of $0.30 \pm 0.01$ and $0.34 \pm 0.01$ for AMBERff 14 and GARF, respectively. Conversely the DSP set, with 36 ligands, exhibits very high and robust Pearson $R$ values of $0.85 \pm 0.00$ and 0.84 \pm 0.00 , respectively, for the two potentials considered. When we consider the LOO MUEs, all of the sets exhibit errors that are less than $1.0 \mathrm{kcal} / \mathrm{mol}$ using either potential. By comparing the Pearson $R$ and MUE values in Table 4 for all 10 protein test sets, we found that the two potentials were in good agreement when the binding affinities were evaluated against the crystal structure binding modes using the $\mathrm{MT}_{\text {ScoreES }}$ protocol.

With the MOE docking program, as shown in Figure 11 and Table 4, the AMBERff14 force field and the GARF energy function showed similar prediction accuracies. For both functions, the $\mathrm{MT}_{\mathrm{ScoreE}}$ protocol generated better or comparable Pearson $R$ coefficients and MUE values compared with the $\mathrm{MT}_{\text {ScoreES }}$ protocol against all protein sets, suggesting that-as with the CASF set-given "good" poses the methods converge well and the MT method itself is quite robust. On the other hand, with the $\mathrm{MT}_{\text {Dock }}$ module in the DivCon Discovery Suite, the AMBERff14 force field and GARF energy function showed different prediction accuracies against the 10 protein families. With AMBERff14 force field, the $\mathrm{MT}_{\text {ScoreE }}$ protocol had better or comparable Pearson $R$ coefficients compared to the $\mathrm{MT}_{\mathrm{ScoreES}}$ protocol against most of the protein sets, except for AChR and GBA3. With the GARF energy function, the $\mathrm{MT}_{\text {ScoreE }}$ protocol showed good ranking performance in all the protein sets, and it especially improved the Pearson $R$ coefficients for the PDPK1 and GBA3 test sets. In a comparison of the MUEs, the AMBERff14 force field outperformed the GARF energy function with the 14-3-3 $\eta$, DHQD, DSP, and Tryp sets, while the GARF energy function generated significantly lower MUEs with the GBA3, BC, and AChE sets.

As depicted in Figure 11, in a comparison of the MOE rigidreceptor docking protocol with the $\mathrm{MT}_{\text {Dock }}$ rigid-receptor protocol, the two potentials showed generally good agreement with one another for the MOE-docked poses. However, as shown in Figure 12, the Pearson $R$ was significantly improved for the PDPK1, GBA3, and BC sets when the three-step $\mathrm{MT}_{\text {Dock }}$ protocol with GARF was used, compared with the two-step $\mathrm{MT}_{\text {ScoreE }}$ with the MOE docker. This would suggest that the binding affinity prediction benefits from the introduction of conformational entropies that are captured in the three-step $\mathrm{MT}_{\mathrm{CS}}$-driven method but not in the two-step method. On the other hand, when the three-step $\mathrm{MT}_{\text {ScoreE }}$ 

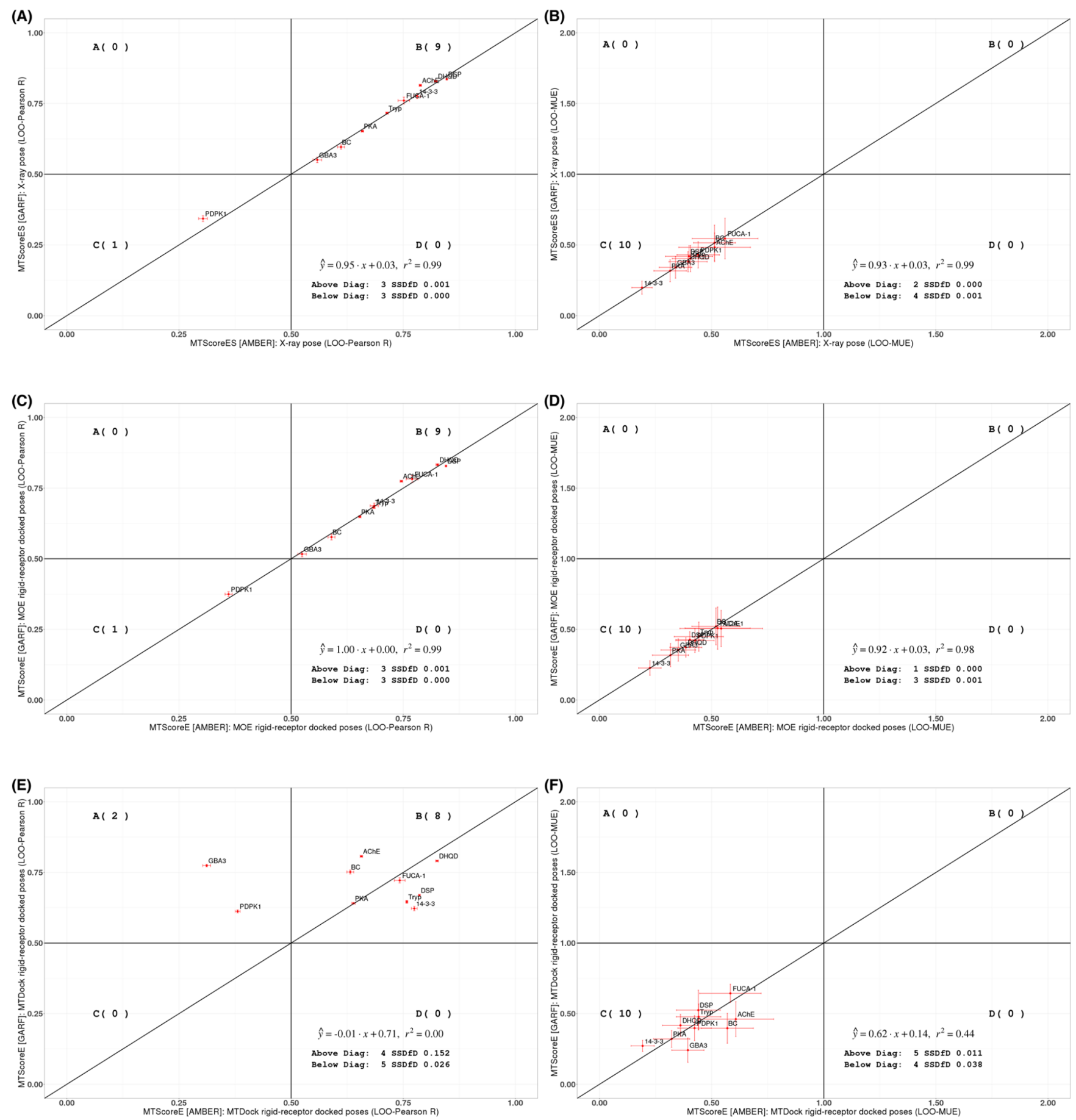

Figure 11. Comparison of Pearson $R$ correlations and MUEs between the GARF and AMBERff14 energy functions obtained using different MT calculation settings and protocols. (A, B) $\mathrm{MT}_{\mathrm{ScoreES}}$ calculations for the two energy functions: (A) Pearson $R$ values; (B) MUE values. (C, D) Calculations with MOE using the $\mathrm{MT}_{\text {ScoreE }}$ protocol for the two potentials: (C) Pearson $R$ values; (D) MUE values. (E, F) Calculations with $\mathrm{MT}_{\text {Dock }}$ using the $\mathrm{MT}_{\text {ScoreE }}$ protocol for the two energy functions: (E) Pearson $R$ values; (F) MUE values.

protocol was used with GARF against the 14-3-3 $\eta$ and DSP sets and with Amberff14 against GBA3, the Pearson $R$ was lower compared with the MOE $\mathrm{MT}_{\text {ScoreE }}$ results, suggesting that in these cases the $\mathrm{MT}_{\mathrm{CS}}$ conformers were inferior to the conformers provided by MOE. Furthermore, the diverging atom:atom pair potential-dependent results we observed with $\mathrm{MT}_{\text {Dock }}$ are attributable to the different $\mathrm{MT}_{\mathrm{CS}}$ conformers generated with the two potentials: while MOE generates the same conformers and the same binding modes regardless of chosen $\mathrm{MT}$ potential, $\mathrm{MT}_{\mathrm{CS}}$ uses the chosen potential to define the target bond lengths, angles, and torsions. ${ }^{59}$ Therefore, the consistent agreement we see between GARF and AMBERff14 in both the HPF and CASF benchmarks when they are challenged with pair-potential-independent MOE poses suggests that the MT method itself is quite robust. That said, as depicted in Figure 12, GARF does appear to exhibit some preference for GARF-generated $\mathrm{MT}_{\mathrm{CS}}$ poses. 

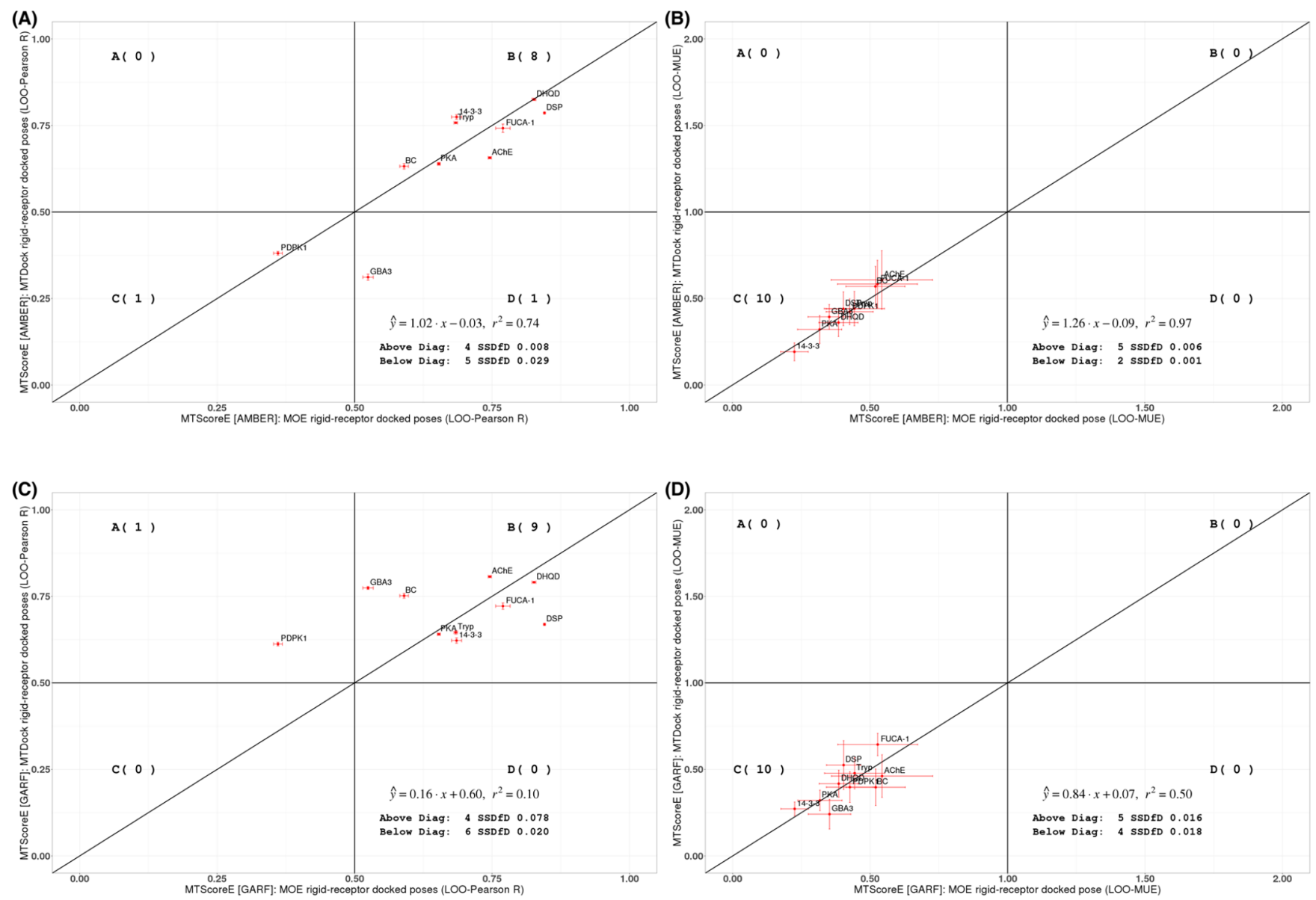

Figure 12. Comparison of Pearson $R$ correlations and MUEs between the GARF and AMBERff14 energy functions using different docking programs in the $\mathrm{MT}_{\text {ScoreE }}$ protocol. (A) Pearson $R$ values for AMBER for $\mathrm{MT}_{\text {Dock }}$ compared to MOE dock. (B) MUE values for AMBER for $\mathrm{MT}_{\text {Dock }}$ compared to MOE dock. (C) Pearson $R$ values for GARF for $\mathrm{MT}_{\text {Dock }}$ compared to MOE dock. (D) MUE values for GARF for MT ${ }_{\text {Dock }}$ compared to MOE dock.

\section{CONCLUSIONS}

Large-scale routine application of computational receptorligand simulation and binding free energy prediction in industrial drug discovery remains a daunting task. Obtaining the proper balance of computational cost and efficiency in molecular energy state sampling is a central problem for this issue. In this paper, we have reported a new approach bringing the "Movable Type" free energy method from a theoretical concept to a functional software package. The current version of the MT software package provides two main free energy workflows: $\mathrm{MT}_{\text {ScoreEs }}$ for fast and simple calculations, which applies only the local partition function sampling regime to a single initial molecular conformation (e.g., the crystal structure as in this work or a structure chosen through other methods by the practitioner in the field), and $\mathrm{MT}_{\mathrm{ScoreE}}$, which is a complete computational protocol including both unbound- and boundstate configurational sampling. Two energy functions, the AMBERff14 force field and the GARF statistical potential function, are also provided as different options for the energy evaluation of the sampled conformations (and though this is beyond the scope of this paper, users may also substitute alternative functions as well through the use of standard parmtop/coord files). The $\mathrm{MT}_{\text {ScoreE }}$ method can be executed in both a "two-step" and a "three-step" workflow, and it can generate its own landscape minima using the built-in $\mathrm{MT}_{\text {Dock }}$ approach or be supplied with binding modes generated through other means (e.g., MOE, GLIDE, etc.). Furthermore, as demonstrated in the present work, the method is also able to characterize not only ligand-side movement/sampling but also protein-side sampling. Future work will build on this support to include multiple apo protein and holo-protein:ligand conformers such as those available from X-ray, cryogenic electron microscopy, and NMR experimental models along with theoretical models and trajectories.

In this paper, these protocol combinations were validated in order to demonstrate the overall robustness of the method. The prediction profile of MT is shown to be remarkably robust, and given good theoretical landscape minima (e.g., reasonably docked poses), clearly the ensemble method is able to do as well as or sometimes better than poses generated through much more expensive means (e.g., X-ray crystallography). Together, these results show that the DivCon Discovery Suite with the MT module is a good option for fast free-energy-based receptor-ligand virtual screening applied to rational drug design studies.

\section{ASSOCIATED CONTENT}

\section{Supporting Information}

The Supporting Information is available free of charge at https://pubs.acs.org/doi/10.1021/acs.jcim.0c00618.

PDB models included in the Homologous Protein Family set (PDF) 


\section{AUTHOR INFORMATION}

\section{Corresponding Author}

Lance M. Westerhoff - QuantumBio Inc., State College, Pennsylvania 16801, United States; 이 orcid.org/0000-00018092-6919; Email: lance@quantumbioinc.com

\section{Authors}

Zheng Zheng - QuantumBio Inc., State College, Pennsylvania 16801, United States; School of Chemistry, Chemical Engineering and Life Science, Wuhan University of Technology,

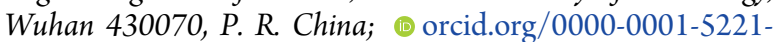
3209

Oleg Y. Borbulevych - QuantumBio Inc., State College, Pennsylvania 16801, United States

Hao Liu - School of Mechanical and Electronic Engineering, Wuhan University of Technology, Wuhan 430070, P. R. China

Jianpeng Deng - School of Chemistry, Chemical Engineering and Life Science, Wuhan University of Technology, Wuhan 430070, P. R. China

Roger I. Martin - QuantumBio Inc., State College, Pennsylvania 16801, United States

Complete contact information is available at:

https://pubs.acs.org/10.1021/acs.jcim.0c00618

\section{Notes}

The authors declare the following competing financial interest(s): One or more of the authors is a QuantumBio Inc. employee, consultant, and/or shareholder.

All of the prepared structural information (including input, output, and associated structures) for both the CASF set and the HPF set is available at the following URL: http:// downloads.quantumbioinc.com/media/tutorials/MT/MTFreeEnergyPaper.tar.bz2.

\section{ACKNOWLEDGMENTS}

The authors wish to acknowledge the continued support of our customers, clients, and collaborators who have provided valuable feedback and protocol suggestions on the Movable Type technology. The authors also acknowledge the continued support of Michigan State University and MSU Technologies for licensing the MovableType technology to QuantumBio Inc. The authors thank Chemical Computing Group (in particular Alain Deschenes, Chris Williams, Paul Labute, and the entire CCG support team) for their continued support with MOE best practices and with the Scientific Vector Language. Finally, the authors thank Nupur Bansal and Kenneth M. Merz, Jr., for their helpful discussions early in the technology transfer process. The research reported in this publication was supported in part by the National Institute of General Medical Sciences of the National Institutes of Health under Small Business Innovative Research (SBIR) Awards R44GM134781, R44GM121162, and R44GM112406. The content is solely the responsibility of the authors and does not necessarily represent the official views of the National Institutes of Health.

\section{REFERENCES}

(1) Paul, S. M.; Mytelka, D. S.; Dunwiddie, C. T.; Persinger, C. C.; Munos, B. H.; Lindborg, S. R.; Schacht, A. L. How to improve R\&D productivity: the pharmaceutical industry's grand challenge. Nat. Rev. Drug Discovery 2010, 9, 203-14.

(2) Dickson, M.; Gagnon, J. P. The cost of new drug discovery and development. Discovery Med. 2004, 4, 172-179.
(3) Muller-Dethlefs, K.; Hobza, P. Noncovalent interactions: A challenge for experiment and theory. Chem. Rev. 2000, 100, 143-167. (4) Riley, K.; Pitoňák, M.; Černý, J.; Hobza, P. On the Structure and Geometry of Biomolecular Binding Motifs (Hydrogen-Bonding, Stacking, $\mathrm{X}-\mathrm{H} \cdots \pi)$ : WFT and DFT Calculations. J. Chem. Theory Comput. 2010, 6, 66-80.

(5) Raha, K.; Peters, M. B.; Wang, B.; Yu, N.; Wollacott, A. M.; Westerhoff, L. M.; Merz, K. M., Jr. The role of quantum mechanics in structure-based drug design. Drug Discovery Today 2007, 12, 725-31.

(6) Kuntz, I. D. Structure-based strategies for drug design and discovery. Science 1992, 257, 1078-82.

(7) Jorgensen, W. L. The many roles of computation in drug discovery. Science 2004, 303, 1813-8.

(8) Jorgensen, W. L. Efficient Drug Lead Discovery and Optimization. Acc. Chem. Res. 2009, 42, 724-733.

(9) Zhang, X.; Gibbs, A. C.; Reynolds, C. H.; Peters, M. B.; Westerhoff, L. M. Quantum mechanical pairwise decomposition analysis of protein kinase B inhibitors: validating a new tool for guiding drug design. J. Chem. Inf. Model. 2010, 50, 651-61.

(10) Diller, D. J.; Humblet, C.; Zhang, X.; Westerhoff, L. M. Computational alanine scanning with linear scaling semiempirical quantum mechanical methods. Proteins: Struct., Funct., Genet. 2010, 78, 2329-37.

(11) Warren, G. L.; Andrews, C. W.; Capelli, A. M.; Clarke, B.; LaLonde, J.; Lambert, M. H.; Lindvall, M.; Nevins, N.; Semus, S. F.; Senger, S.; Tedesco, G.; Wall, I. D.; Woolven, J. M.; Peishoff, C. E.; Head, M. S. A critical assessment of docking programs and scoring functions. J. Med. Chem. 2006, 49, 5912-5931.

(12) Moustakas, D. T.; Lang, P. T.; Pegg, S.; Pettersen, E.; Kuntz, I. D.; Brooijmans, N.; Rizzo, R. C. Development and validation of a modular, extensible docking program: DOCK 5. J. Comput.-Aided Mol. Des. 2006, 20, 601-619.

(13) Hartshorn, M. J.; Verdonk, M. L.; Chessari, G.; Brewerton, S. C.; Mooij, W. T. M.; Mortenson, P. N.; Murray, C. W. Diverse, highquality test set for the validation of protein-ligand docking performance. J. Med. Chem. 2007, 50, 726-741.

(14) Verdonk, M. L.; Cole, J. C.; Hartshorn, M. J.; Murray, C. W.; Taylor, R. D. Improved protein-ligand docking using GOLD. Proteins: Struct., Funct., Genet. 2003, 52, 609-623.

(15) Schneider, G. Virtual screening: an endless staircase? Nat. Rev. Drug Discovery 2010, 9, 273-6.

(16) Michel, J.; Essex, J. W. Prediction of protein-ligand binding affinity by free energy simulations: assumptions, pitfalls and expectations. J. Comput.-Aided Mol. Des. 2010, 24, 639-58.

(17) Kolb, P.; Irwin, J. J. Docking Screens: Right for the Right Reasons? Curr. Top. Med. Chem. 2009, 9, 755-770.

(18) Deng, Y. Q.; Roux, B. Computations of Standard Binding Free Energies with Molecular Dynamics Simulations. J. Phys. Chem. B 2009, 113, 2234-2246.

(19) Gallicchio, E.; Levy, R. M. Advances in all atom sampling methods for modeling protein-ligand binding affinities. Curr. Opin. Struct. Biol. 2011, 21, 161-166.

(20) Karplus, M. Dynamical aspects of molecular recognition. J. Mol. Recognit. 2010, 23, 102-4.

(21) Mobley, D. L.; Dill, K. A. Binding of small-molecule ligands to proteins: "what you see" is not always "what you get". Structure 2009, 17, 489-498.

(22) Young, T.; Abel, R.; Kim, B.; Berne, B. J.; Friesner, R. A. Motifs for molecular recognition exploiting hydrophobic enclosure in protein-ligand binding. Proc. Natl. Acad. Sci. U. S. A. 2007, 104, $808-813$.

(23) Luccarelli, J.; Michel, J.; Tirado-Rives, J.; Jorgensen, W. L. Effects of Water Placement on Predictions of Binding Affinities for p38alpha MAP Kinase Inhibitors. J. Chem. Theory Comput. 2010, 6, $3850-3856$

(24) Michel, J.; Tirado-Rives, J.; Jorgensen, W. L. Energetics of displacing water molecules from protein binding sites: consequences for ligand optimization. J. Am. Chem. Soc. 2009, 131, 15403-11. 
(25) Martin, Y. C. Let's not forget tautomers. J. Comput.-Aided Mol. Des. 2009, 23, 693-704.

(26) Pospisil, P.; Ballmer, P.; Scapozza, L.; Folkers, G. Tautomerism in computer-aided drug design. J. Recept. Signal Transduction Res. 2003, 23, 361-71.

(27) Tirado-Rives, J.; Jorgensen, W. L. Contribution of conformer focusing to the uncertainty in predicting free energies for proteinligand binding. J. Med. Chem. 2006, 49, 5880-5884.

(28) Merz, K. M. Limits of Free Energy Computation for ProteinLigand Interactions. J. Chem. Theory Comput. 2010, 6, 1769-1776.

(29) Faver, J. C.; Benson, M. L.; He, X.; Roberts, B. P.; Wang, B.; Marshall, M. S.; Kennedy, M. R.; Sherrill, C. D.; Merz, K. M., Jr. Formal Estimation of Errors in Computed Absolute Interaction Energies of Protein-ligand Complexes. J. Chem. Theory Comput. 2011, 7, 790-797.

(30) Nissink, J. W.; Murray, C.; Hartshorn, M.; Verdonk, M. L.; Cole, J. C.; Taylor, R. A new test set for validating predictions of protein-ligand interaction. Proteins: Struct., Funct., Genet. 2002, 49, 457-71.

(31) Perola, E.; Charifson, P. S. Conformational analysis of drug-like molecules bound to proteins: an extensive study of ligand reorganization upon binding. J. Med. Chem. 2004, 47, 2499-510.

(32) Lim, N. M.; Wang, L.; Abel, R.; Mobley, D. L. Sensitivity in Binding Free Energies Due to Protein Reorganization. J. Chem. Theory Comput. 2016, 12, 4620-31.

(33) Teague, S. J. Implications of protein flexibility for drug discovery. Nat. Rev. Drug Discovery 2003, 2, 527-41.

(34) Lill, M. A. Efficient incorporation of protein flexibility and dynamics into molecular docking simulations. Biochemistry 2011,50, 6157-69.

(35) FEP+; Schrödinger, LLC: New York.

(36) Salomon-Ferrer, R.; Case, D. A.; Walker, R. C. An overview of the Amber biomolecular simulation package. Wiley Interdiscip. Rev.: Comput. Mol. Sci. 2013, 3, 198-210.

(37) Case, D. A.; Ben-Shalom, I. Y.; Brozell, S. R.; Cerutti, T. E.; Cheatham, T. E.; Cruzeiro, V. W. D.; Darden, T. A.; Duke, R. E.; Ghoreishi, D.; Gilson, M. K.; Gohlke, H.; Goetz, A. W.; Greene, D.; Harris, R.; Homeyer, N.; Izadi, S.; Kovalenko, A.; Kurtzman, T.; Lee, T. S.; LeGrand, S.; Li, P.; Lin, C.; Liu, J.; Luchko, T.; Luo, R.; Mermelstein, D. J.; Merz, K. M.; Miao, Y.; Monard, G.; Nguyen, C.; Nguyen, H.; Omelyan, I.; Onufriev, A.; Pan, F.; Qi, R.; Roe, D. R.; Roitberg, A.; Sagui, C.; Schott-Verdugo, S.; Shen, J.; Simmerling, C. L.; Smith, J.; Salomon-Ferrer, R.; Swails, J.; Walker, R. C.; Wang, J.; Wei, H.; Wolf, R. M.; Wu, X.; Xiao, L.; York, D. M.; Kollman, P. A. AMBER 2018; University of California: San Francisco, 2018.

(38) Bea, I.; Cervello, E.; Kollman, P. A.; Jaime, C. Molecular recognition by beta-cyclodextrin derivatives: FEP vs MM/PBSA study. Comb. Chem. High Throughput Screening 2001, 4, 605-11.

(39) Kuhn, B.; Kollman, P. A. Binding of a diverse set of ligands to avidin and streptavidin: an accurate quantitative prediction of their relative affinities by a combination of molecular mechanics and continuum solvent models. J. Med. Chem. 2000, 43, 3786-3791.

(40) Honig, B.; Nicholls, A. Classical electrostatics in biology and chemistry. Science 1995, 268, 1144-9.

(41) Masukawa, K. M.; Kollman, P. A.; Kuntz, I. D. Investigation of neuraminidase-substrate recognition using molecular dynamics and free energy calculations. J. Med. Chem. 2003, 46, 5628-37.

(42) Wallnoefer, H. G.; Liedl, K. R.; Fox, T. A challenging system: free energy prediction for factor Xa. J. Comput. Chem. 2011, 32, $1743-52$.

(43) Aqvist, J.; Medina, C.; Samuelsson, J. E. A new method for predicting binding affinity in computer-aided drug design. Protein Eng., Des. Sel. 1994, 7, 385-91.

(44) Liu, P.; Kim, B.; Friesner, R. A.; Berne, B. J. Replica exchange with solute tempering: a method for sampling biological systems in explicit water. Proc. Natl. Acad. Sci. U. S. A. 2005, 102, 13749-54.

(45) Wang, L.; Deng, Y.; Knight, J. L.; Wu, Y.; Kim, B.; Sherman, W.; Shelley, J. C.; Lin, T.; Abel, R. Modeling Local Structural Rearrangements Using FEP/REST: Application to Relative Binding
Affinity Predictions of CDK2 Inhibitors. J. Chem. Theory Comput. 2013, 9, 1282-93.

(46) Wang, L.; Wu, Y.; Deng, Y.; Kim, B.; Pierce, L.; Krilov, G.; Lupyan, D.; Robinson, S.; Dahlgren, M. K.; Greenwood, J.; Romero, D. L.; Masse, C.; Knight, J. L.; Steinbrecher, T.; Beuming, T.; Damm, W.; Harder, E.; Sherman, W.; Brewer, M.; Wester, R.; Murcko, M.; Frye, L.; Farid, R.; Lin, T.; Mobley, D. L.; Jorgensen, W. L.; Berne, B. J.; Friesner, R. A.; Abel, R. Accurate and reliable prediction of relative ligand binding potency in prospective drug discovery by way of a modern free-energy calculation protocol and force field. J. Am. Chem. Soc. 2015, 137, 2695-703.

(47) Gilson, M. K.; Zhou, H. X. Calculation of protein-ligand binding affinities. Annu. Rev. Biophys. Biomol. Struct. 2007, 36, 21-42.

(48) Chodera, J. D.; Mobley, D. L.; Shirts, M. R.; Dixon, R. W.; Branson, K.; Pande, V. S. Alchemical free energy methods for drug discovery: progress and challenges. Curr. Opin. Struct. Biol. 2011, 21, $150-160$.

(49) Colizzi, F.; Perozzo, R.; Scapozza, L.; Recanatini, M.; Cavalli, A. Single-Molecule Pulling Simulations Can Discern Active from Inactive Enzyme Inhibitors. J. Am. Chem. Soc. 2010, 132, 7361-7371.

(50) Fidelak, J.; Juraszek, J.; Branduardi, D.; Bianciotto, M.; Gervasio, F. L. Free-Energy-Based Methods for Binding Profile Determination in a Congeneric Series of CDK2 Inhibitors. J. Phys. Chem. B 2010, 114, 9516-9524.

(51) Doudou, S.; Sharma, R.; Henchman, R. H.; Sheppard, D. W.; Burton, N. A. Inhibitors of PIM-1 Kinase: A Computational Analysis of the Binding Free Energies of a Range of Imidazo [1,2-b] Pyridazines. J. Chem. Inf. Model. 2010, 50, 368-379.

(52) Doudou, S.; Burton, N. A.; Henchman, R. H. Standard Free Energy of Binding from a One-Dimensional Potential of Mean Force. J. Chem. Theory Comput. 2009, 5, 909-918.

(53) Buch, I.; Harvey, M. J.; Giorgino, T.; Anderson, D. P.; De Fabritiis, G. High-Throughput All-Atom Molecular Dynamics Simulations Using Distributed Computing. J. Chem. Inf. Model. 2010, 50, 397-403.

(54) Le, L.; Lee, E. H.; Hardy, D. J.; Truong, T. N.; Schulten, K. Molecular Dynamics Simulations Suggest that Electrostatic Funnel Directs Binding of Tamiflu to Influenza N1 Neuraminidases. PLoS Comput. Biol. 2010, 6, e1000939.

(55) Zheng, Z.; Ucisik, M. N.; Merz, K. M. The Movable Type Method Applied to Protein-Ligand Binding. J. Chem. Theory Comput. 2013, 9, 5526-5538.

(56) Zheng, Z.; Merz, K. M., Jr. Movable type method applied to protein-ligand binding. US $20160350474 \mathrm{A1}, 2016$.

(57) Bansal, N.; Zheng, Z.; Merz, K. M., Jr. Incorporation of side chain flexibility into protein binding pockets using MTflex. Bioorg. Med. Chem. 2016, 24, 4978-4987.

(58) Bansal, N.; Zheng, Z.; Song, L. F.; Pei, J.; Merz, K. M., Jr. The Role of the Active Site Flap in Streptavidin/Biotin Complex Formation. J. Am. Chem. Soc. 2018, 140, 5434-5446.

(59) Pan, L. L.; Zheng, Z.; Wang, T.; Merz, K. M., Jr. Free EnergyBased Conformational Search Algorithm Using the Movable Type Sampling Method. J. Chem. Theory Comput. 2015, 11, 5853-64.

(60) Zhong, H. A.; Santos, E. M.; Vasileiou, C.; Zheng, Z.; Geiger, J. H.; Borhan, B.; Merz, K. M., Jr. Free-Energy-Based Protein Design: Re-Engineering Cellular Retinoic Acid Binding Protein II Assisted by the Moveable-Type Approach. J. Am. Chem. Soc. 2018, 140, 34833486.

(61) Zheng, Z.; Wang, T.; Li, P.; Merz, K. M. KECSA-Movable Type Implicit Solvation Model (KMTISM). J. Chem. Theory Comput. 2015, 11, 667-682.

(62) Wang, B.; Westerhoff, L. M.; Merz, K. M. A critical assessment of the performance of protein-ligand scoring functions based on NMR chemical shift perturbations. J. Med. Chem. 2007, 50, 51285134.

(63) Borbulevych, O.; Martin, R. I.; Westerhoff, L. M. Highthroughput quantum-mechanics/molecular-mechanics (ONIOM) macromolecular crystallographic refinement with PHENIX/DivCon: 
the impact of mixed Hamiltonian methods on ligand and protein structure. Acta Crystallogr., Sect. D. Struct. Biol. 2018, 74, 1063-1077. (64) Borbulevych, O.; Martin, R. I.; Tickle, I. J.; Westerhoff, L. M. XModeScore: a novel method for accurate protonation/tautomerstate determination using quantum-mechanically driven macromolecular X-ray crystallographic refinement. Acta Crystallogr., Sect. D: Struct. Biol. 2016, 72, 586-98.

(65) Borbulevych, O. Y.; Plumley, J. A.; Martin, R. I.; Merz, K. M., Jr.; Westerhoff, L. M. Accurate macromolecular crystallographic refinement: incorporation of the linear scaling, semiempirical quantum-mechanics program DivCon into the PHENIX refinement package. Acta Crystallogr., Sect. D: Biol. Crystallogr. 2014, 70, 123347.

(66) Shaw, D. E.; Deneroff, M. M.; Dror, R. O.; Kuskin, J. S.; Larson, R. H.; Salmon, J. K.; Young, C.; Batson, B.; Bowers, K. J.; Chao, J. C.; Eastwood, M. P.; Gagliardo, J.; Grossman, J. P.; Ho, C. R.; Ierardi, D. J.; Kolossváry, I.; Klepeis, J. L.; Layman, T.; McLeavey, C.; Moraes, M. A.; Mueller, R.; Priest, E. C.; Shan, Y.; Spengler, J.; Theobald, M.; Towles, B.; Wang, S. C. Anton, a special-purpose machine for molecular dynamics simulation. Commun. ACM 2008, 51, 91-97.

(67) Lee, T.-S.; Cerutti, D. S.; Mermelstein, D.; Lin, C.; LeGrand, S.; Giese, T. J.; Roitberg, A.; Case, D. A.; Walker, R. C.; York, D. M. GPU-Accelerated Molecular Dynamics and Free Energy Methods in Amber18: Performance Enhancements and New Features. J. Chem. Inf. Model. 2018, 58, 2043-2050.

(68) Lee, T.-S.; Hu, Y.; Sherborne, B.; Guo, Z.; York, D. M. Toward Fast and Accurate Binding Affinity Prediction with pmemdGTI: An Efficient Implementation of GPU-Accelerated Thermodynamic Integration. J. Chem. Theory Comput. 2017, 13, 3077-3084.

(69) Mermelstein, D. J.; Lin, C.; Nelson, G.; Kretsch, R.; McCammon, J. A.; Walker, R. C. Fast and flexible gpu accelerated binding free energy calculations within the amber molecular dynamics package. J. Comput. Chem. 2018, 39, 1354-1358.

(70) Zheng, Z.; Pei, J.; Bansal, N.; Liu, H.; Song, L. F.; Merz, K. M., Jr. Generation of Pairwise Potentials Using Multidimensional Data Mining. J. Chem. Theory Comput. 2018, 14, 5045-5067.

(71) Maier, J. A.; Martinez, C.; Kasavajhala, K.; Wickstrom, L.; Hauser, K. E.; Simmerling, C. ff14SB: Improving the Accuracy of Protein Side Chain and Backbone Parameters from ff99SB. J. Chem. Theory Comput. 2015, 11, 3696-3713.

(72) Fuhrmann, J.; Rurainski, A.; Lenhof, H.-P.; Neumann, D. A new method for the gradient-based optimization of molecular complexes. J. Comput. Chem. 2009, 30, 1371-1378.

(73) Labute, P. Protonate3D: assignment of ionization states and hydrogen coordinates to macromolecular structures. Proteins: Struct., Funct., Genet. 2009, 75, 187-205.

(74) Corbeil, C. R.; Williams, C. I.; Labute, P. Variability in docking success rates due to dataset preparation. J. Comput.-Aided Mol. Des. 2012, 26, 775-786.

(75) Sachs, L. Applied Statistics: A Handbook of Techniques; Springer: New York, 1984.

(76) Su, M.; Yang, Q.; Du, Y.; Feng, G.; Liu, Z.; Li, Y.; Wang, R. Comparative Assessment of Scoring Functions: The CASF-2016 Update. J. Chem. Inf. Model. 2019, 59, 895-913.

(77) Liu, Z.; Li, Y.; Han, L.; Li, J.; Liu, J.; Zhao, Z.; Nie, W.; Liu, Y.; Wang, R. PDB-wide collection of binding data: current status of the PDBbind database. Bioinformatics 2015, 31, 405-412.

(78) Sherman, W.; Day, T.; Jacobson, M. P.; Friesner, R. A.; Farid, R. Novel procedure for modeling ligand/receptor induced fit effects. $J$. Med. Chem. 2006, 49, 534-53.

(79) Du, Q.; Qian, Y.; Yao, X.; Xue, W. Elucidating the tight-binding mechanism of two oral anticoagulants to factor $\mathrm{Xa}$ by using inducedfit docking and molecular dynamics simulation. J. Biomol. Struct. Dyn. 2020, 38, 625-633.

(80) Wang, Y.; Sun, Y.; Cao, R.; Liu, D.; Xie, Y.; Li, L.; Qi, X.; Huang, N. In Silico Identification of a Novel Hinge-Binding Scaffold for Kinase Inhibitor Discovery. J. Med. Chem. 2017, 60, 8552-8564.

(81) Ghose, A. K.; Herbertz, T.; Pippin, D. A.; Salvino, J. M.; Mallamo, J. P. Knowledge based prediction of ligand binding modes and rational inhibitor design for kinase drug discovery. J. Med. Chem. 2008, 51, 5149-71.

(82) Zhong, H.; Tran, L. M.; Stang, J. L. Induced-fit docking studies of the active and inactive states of protein tyrosine kinases. J. Mol. Graphics Modell. 2009, 28, 336-46. 